\title{
An Analytical Theory of the Signal-to-Noise Ratio of Hall Plates with Four Contacts and a Single Mirror Symmetry
}

\author{
Udo Ausserlechner \\ Sense and Control, Infineon Technologies AG, Villach, Austria \\ Email: udo.ausserlechner@infineon.com
}

How to cite this paper: Ausserlechner, U. (2018) An Analytical Theory of the Signal-to-Noise Ratio of Hall Plates with Four Contacts and a Single Mirror Symmetry. Journal of Applied Mathematics and Physics, 6, 2032-2066.

https://doi.org/10.4236/jamp.2018.610174

Received: September 11, 2018

Accepted: October 21, 2018

Published: October 24, 2018

Copyright $\odot 2018$ by author and Scientific Research Publishing Inc. This work is licensed under the Creative Commons Attribution International License (CC BY 4.0).

http://creativecommons.org/licenses/by/4.0/

\begin{abstract}
This work gives an analytical theory of the signal-to-thermal-noise ratio (SNR) of classical Hall plates with four contacts at small magnetic field. In contrast to previous works, the symmetry of the Hall plates is reduced to only a single mirror axis, whereby the average of potentials of the two output contacts off this mirror axis differs from the average of potentials at the two supply contacts on the mirror axis, i.e. the output common mode differs from $50 \%$. Surprisingly, at fixed power dissipated in the Hall plate, the maximum achievable SNR is only $9 \%$ smaller for output common modes of $30 \%$ and $70 \%$ when compared to the overall optimum at output common modes of $50 \%$. The theory is applied to Vertical Hall effect devices with three contacts on the top surface and one contact being the buried layer in a silicon BiCMOS process. Geometries are found with large contacts and only a moderate loss in SNR.
\end{abstract}

\section{Keywords}

Hall Plate, Vertical Hall Effect Device, Geometry Factor, Conformal Mapping, Signal to Noise Ratio, Single Mirror Symmetry, Equivalent Resistor Circuit, Common Mode Potential

\section{Introduction}

This paper focuses on impedances, magnetic sensitivity, and thermal noise of Hall plates with four contacts at small magnetic field. The specific question we want to answer is, how much does the signal to noise ratio (SNR) deteriorate for devices with reduced symmetry. This is a matter of layout and shape-not of technology. Conventional Hall plates are $90^{\circ}$ symmetric (Figure 1(a)) or they 


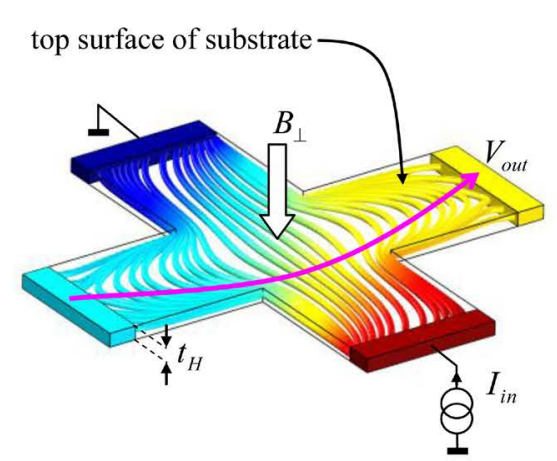

(a)

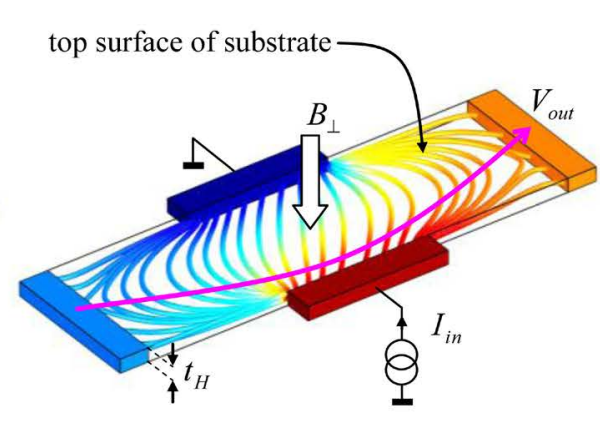

(b)

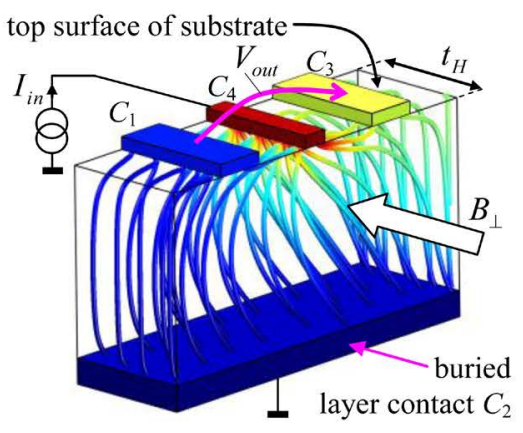

(c)

Figure 1. Hall plates with four contacts and (a) with $90^{\circ}$ symmetry, (b) with two perpendicular mirror symmetries, (c) vertical Hall effect device with a single mirror symmetry. The theory in this paper applies to all these topologies. There, the current flow lines span a plane which is perpendicular to the detectable magnetic field $B_{\perp}$ : in ((a), (b)) this plane is the top surface of the substrate; in (c) this plane intersects the top surface of the substrate at $90^{\circ}$. The thickness $t_{H}$ of the device is defined along the direction of $B_{\perp}$. The figures show the current flow lines for a large Hall angle of $45^{\circ}$, and the colour coding gives the electric potential (red means positive potential, blue means negative potential).

have at least two perpendicular mirror symmetries (Figure 1(b)), both of which can have maximum SNR for properly sized contacts [1]. Yet, for Vertical Hall effect devices, one is forced to use shapes having only a single mirror symmetry (Figure 1(c)) [2] [3]. These devices are able to detect the magnetic field parallel to the main surface of a chip, whereas conventional Hall plates detect the magnetic field orthogonal to the main chip surface. Vertical Hall effect devices are attractive in cost sensitive mass markets for linear [4], rotational [5], and 3D position sensing in automotive, industrial, and home appliance applications. Compared to competing XMR technologies (like tunneling magneto-resistance) they can be cheaper in manufacturing and end of line testing, more robust against environmental conditions, more linear versus magnetic field, they have no saturation at reasonable flux densities, no hysteresis and no crosstalk between orthogonal magnetic field components ${ }^{1}$, and they offer still lower zero point errors when operated in spinning current schemes [6] —even for wide analogue bandwidths up to $400 \mathrm{kHz}$ [7]. The drawback of silicon Vertical Hall effect devices against XMRs is limited SNR, even though their low frequency noise is inherently removed by the spinning scheme [8].

Hall plates with four extended contacts and four-fold symmetry with equal input and output resistance $R_{i n}=R_{\text {out }}$ are discussed in [9] [10] [11] [12] [13]. Their equivalent resistor circuit (ERC) at vanishing magnetic field is shown in Figure 2(a). It consists of two resistors $R_{H}, R_{D}$-thus, it has two electrical degrees of freedom DoF: one DoF can be attributed to the sheet resistance $R_{\mathrm{sh}}=\left(\sigma_{0} t_{H}\right)^{-1}$ and the second DoF can be attributed to the effective number of squares $\lambda_{i n}=R_{i n} / R_{\mathrm{sh}}$ with $R_{i n}=R_{H} \|\left(2 R_{D}\right) \quad$ (with the specific electric conductivity $\sigma_{0}$ and the Hall plate thickness $t_{H}$; the two vertical strokes denote parallel connection of resistors). The sheet resistance describes the thickness of

${ }^{1}$ Strictly speaking the crosstalk of orthogonal magnetic field components vanishes only for zero mechanical shear stress. 


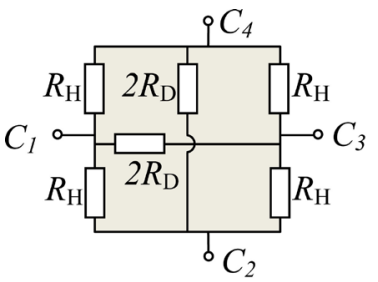

(a)

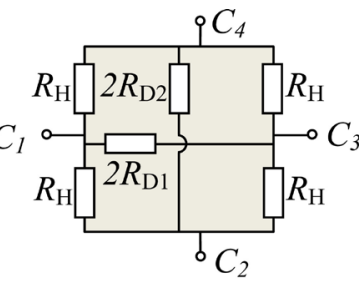

(b)

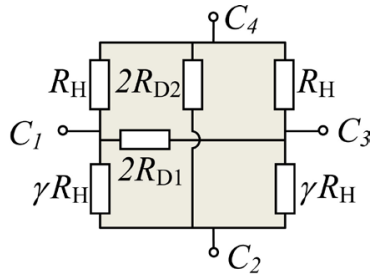

(c)

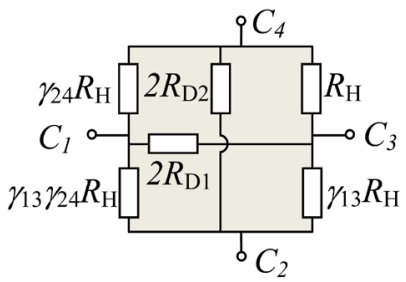

(d)

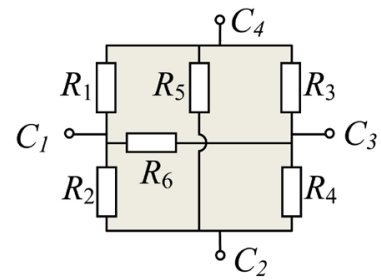

(e)

Figure 2. Equivalent Resistor Circuits (ERCs) of Hall plates with four contacts at zero magnetic field. Devices (a) with $90^{\circ}$ symmetry (b) with two perpendicular mirror symmetry axes along contacts $C_{2}-C_{4}$ and $C_{1}-C_{3}$, yet with different resistances between $C_{1}-C_{3}$ and $C_{2}-C_{4}$ (c) with a single mirror symmetry along contacts $C_{2}-C_{4}$ (d) with no mirror symmetry but zero Hall-output voltage at zero magnetic field for current flow between $C_{1}-C_{3}$ and $C_{2}-C_{4}(\mathrm{e})$ with no symmetry at all.

the Hall plate, which is an out-of-plane parameter. Conversely, $\lambda_{i n}$ describes the lateral geometry of the Hall plate in the plane of the current flow. $\lambda_{i n}$ is an in-plane parameter. Although Vertical Hall effect devices of Figure 1(c) have different orientations than Hall plates of Figure 1(a) \& Figure 1(b), the plane which we refer to is orthogonal to the detectable magnetic field $B_{\perp}$ in both cases. $\lambda_{i n}$ is a ratio of two parameters $R_{i n}, R_{\mathrm{sh}}$ which are accessible to electrical measurements: $R_{i n}$ is simply measured with an Ohm-meter and $R_{\mathrm{sh}}$ is measured according to a generalization of van der Pauw's method [10] [11]. Therefore, $\lambda_{i n}$ links the in-plane geometry of the Hall plate with electrically measurable quantities. However, this link is not a simple ratio of length over width of the Hall plate, except for the case of rectangular plates with flush input contacts and point-sized output contacts [14].

The magnetic sensitivity of a Hall plate at weak magnetic field is defined as change in output voltage $V_{\text {out }}$ per change in flux density $B_{\perp}$ orthogonal to the Hall plate [1] [2] [13]

$$
S_{0}=\lim _{B_{\perp} \rightarrow 0} \frac{\mathrm{d} V_{\text {out }}}{\mathrm{d} B_{\perp}}=\mu_{H} \frac{G_{H 0}}{\lambda_{\text {in }}} V_{\text {in }}=\mu_{H} R_{\mathrm{sh}} G_{H 0} I_{\text {in }}
$$

with the Hall mobility $\mu_{H}$, the Hall input or supply voltage $V_{i n}$, and the Hall input or supply current $I_{i n}$. The low field Hall geometry factor $G_{H 0}$ depends only on the in-plane geometry of the Hall plate, just like $\lambda_{i n}$ does. It holds $0 \leq G_{H 0} \leq 1 . G_{H 0}$ can be expressed as a function of geometrical parameters of the Hall plate, but it can also be expressed more generally as a function of the dimensionless DoFs. Thus, for a symmetric Hall plate with $R_{\text {in }}=R_{\text {out }}$ the Hall geometry factor $G_{H 0}$ is a function of the in-plane parameter $\lambda_{i n}$, not of the 
out-of-plane parameter $R_{\mathrm{sh}}$ [13].

In [1] [14] [15] we also discussed Hall plates with four contacts and two perpendicular mirror symmetries, yet with different input and output resistances. Their common mode output voltages (along both diagonals $C_{1}-C_{3}$ and $C_{2}-C_{4}$, respectively) are at half of the input voltage: $\left(V_{1}+V_{3}\right) / 2=\left(V_{2}+V_{4}\right) / 2$ for current flowing between contacts $C_{2}$ and $C_{4}$, or between contacts $C_{1}$ and $C_{3}$. Thereby, the potential at contact $C_{n}$ is denoted by $V_{n}$ for $n=1,2,3,4$. The ERC of these Hall plates has three resistances $R_{H}, R_{D 1}, R_{D 2}$ as in Figure 2(b). These devices have three DoF: the sheet resistance $R_{\mathrm{sh}}$ and the effective number of squares for both input and output resistances $\lambda_{\text {in }}=R_{\text {in }} / R_{\mathrm{sh}}, \lambda_{\text {out }}=R_{\text {out }} / R_{\mathrm{sh}}$. Many devices can be obtained by conformal transformation on one arbitrary device, and they all have the same ERC, the same $\lambda_{\text {in }}, \lambda_{\text {out }}, R_{\mathrm{sh}}$, and the same magnetic sensitivity $S_{0}$. The Hall-geometry factor is a function of the in-plane DoFs only $G_{H 0}=G_{H 0}\left(\lambda_{\text {in }}, \lambda_{\text {out }}\right)$ (see Fig. 2 in [1] or Fig. 7b in [14]).

The topic of this paper is Hall plates with single mirror symmetry whereby the symmetry axis goes through the centers of two opposite contacts $C_{1}$ and $C_{3}$ like in Figure 1(c). These devices have four degrees of freedom: sheet resistance $R_{\text {sh }}, \ell / W, b / \ell, s / \ell$ (see Figure $3(\mathrm{a})$ ) or alternatively $R_{\mathrm{sh}}, \lambda_{f}, \lambda_{p}, \mathrm{~cm}$. The new parameter $\mathrm{cm}$ is called the common mode and it is defined by

$$
c m=\frac{\left(V_{1}+V_{3}\right) / 2-V_{2}}{V_{4}-V_{2}}
$$

for current flowing from $C_{4}$ to $C_{2}$. Generally, it holds $0 \leq \mathrm{cm} \leq 1$. Devices with two perpendicular mirror symmetries have $\mathrm{cm}=1 / 2$. The ERC of devices with only a single mirror symmetry has six resistors with four resistances:

$R_{H}, \gamma R_{H}, R_{D 1}, R_{D 2}$ with $c m=\gamma /(1+\gamma), \lambda_{f} R_{\mathrm{sh}}=R_{H}(1+\gamma) / 2 \|\left(2 R_{D 2}\right)$, $\lambda_{p} R_{\text {sh }}=\left(2 R_{H} \gamma\right)\left\|\left(2 R_{H}\right)\right\|\left(2 R_{D 1}\right)$. The low field Hall-geometry factor is a function of three in-plane parameters $G_{H 0}=G_{H 0}\left(\lambda_{f}, \lambda_{p}, \mathrm{~cm}\right)$.

Amongst all useful Hall plates with zero output signal at zero magnetic field there are some with no mirror symmetry at all (see Figure 2(d)). They have arbitrary common mode for both pairs of output contacts, which gives a total of five degrees of freedom: $R_{\mathrm{sh}}, \lambda_{f}, \lambda_{p}, \mathrm{~cm}_{13}, \mathrm{~cm}_{24}$. Their ERC has six different resistors yet with the additional constraint that the ratio of two neighboring resistors in the H-bridge-portion is identical to the ratio of the other two resistors in the H-bridge-portion: $R_{H}, \gamma_{13} R_{H}, \gamma_{24} R_{H}, \gamma_{13} \gamma_{24} R_{H}, R_{D 1}, R_{D 2}$ with $c m_{13}=\gamma_{13} /\left(1+\gamma_{13}\right), \quad c m_{24}=\gamma_{24} /\left(1+\gamma_{24}\right)$, $\lambda_{f} R_{\mathrm{sh}}=\gamma_{24}\left(1+\gamma_{13}\right) R_{H}\left\|\left(1+\gamma_{13}\right) R_{H}\right\|\left(2 R_{D 2}\right)$, $\lambda_{p} R_{\mathrm{sh}}=\gamma_{13}\left(1+\gamma_{24}\right) R_{H}\left\|\left(1+\gamma_{24}\right) R_{H}\right\|\left(2 R_{D 1}\right)$. The low field Hall-geometry factor $G_{H 0}$ is a function of the four in-plane parameters $\lambda_{f}, \lambda_{p}, \mathrm{~cm}_{13}, \mathrm{~cm}_{24}$.

In the most general case a resistive device with four terminals has an ERC composed of $3+2+1=6$ resistors: between each couple of terminals there is one resistor, as was shown in [16] (see Figure 2(e)). The six resistors correspond to six DoF: the sheet resistance and five in-plane geometrical parameters (see also page 92 in [17]). The low field Hall-geometry factor is a function of these five 
in-plane parameters. On the other hand, we can normalize all resistances by the sheet resistance, which gives $R_{1} / R_{\mathrm{sh}}, R_{2} / R_{\mathrm{sh}}, \cdots, R_{6} / R_{\mathrm{sh}}$. This set of six values covers only five DoF, because it does not cover $R_{\mathrm{sh}}$ any more. In other words, one of them must be a function of the others: $R_{1} / R_{\mathrm{sh}}=f\left(R_{2} / R_{\mathrm{sh}}, \cdots, R_{6} / R_{\mathrm{sh}}\right)$. If we measure $R_{1}, R_{2}, \cdots, R_{6}$ we can solve this implicit equation numerically for $R_{\mathrm{sh}}$-this is a generalization of van der Pauw's measurement of sheet resistance for devices with extended contacts: The sheet resistance is a function of resistances of the ERC.

$$
R_{\mathrm{sh}}=R_{\mathrm{sh}}\left(R_{1}, R_{2}, \cdots, R_{6}\right)
$$

Moreover, if we normalize $R_{2} / R_{\mathrm{sh}}, \cdots, R_{6} / R_{\mathrm{sh}}$ by $R_{1} / R_{\mathrm{sh}} \quad$ we do not lose any DoF. Therefore, the set

$$
\left\{\frac{R_{2} / R_{\mathrm{sh}}}{R_{1} / R_{\mathrm{sh}}}, \frac{R_{3} / R_{\mathrm{sh}}}{R_{1} / R_{\mathrm{sh}}}, \cdots, \frac{R_{6} / R_{\mathrm{sh}}}{R_{1} / R_{\mathrm{sh}}}\right\}=\left\{\frac{R_{2}}{R_{1}}, \frac{R_{3}}{R_{1}}, \cdots, \frac{R_{6}}{R_{1}}\right\}
$$

also covers the five in-plane DoF and finally it holds

$$
G_{H 0}=G_{H 0}\left(R_{2} / R_{1}, R_{3} / R_{1}, \cdots, R_{6} / R_{1}\right) .
$$

The low field Hall geometry factor is a function of ratios of resistances of the $E R C$. Inserting (3) and (5) into (1) leads to the remarkable conclusion that at small Hall angles the output voltage is fully determined by the ERC, the Hall angle, and the Hall supply current or voltage. We do not need any information on the geometry of the Hall plate. The electrical parameters of the ERC fully determine the sensitivity of the Hall plate output signal with respect to changes in the Hall angle. A similar conclusion was drawn in [18], but there the authors did not link the resistances to the ERC.

The rest of the paper mainly elaborates on the relation $G_{H 0}=G_{H 0}\left(\lambda_{f}, \lambda_{p}, \mathrm{~cm}\right)$ for Hall plates with four contacts of finite size and only one mirror symmetry having ERCs like in Figure 2(c). The author is not aware of any published work about analytical calculations on the magnetic sensitivity of Hall plates of this reduced type of symmetry, although there are a few prior works on entirely asymmetric Hall devices [18] [19]. Conformal mapping was also applied to Vertical Hall devices with five contacts and single mirror symmetry [20]. All these papers compute the Hall signal as a function of geometrical parameters, yet they do not relate the Hall geometry factor to electrical parameters $\lambda_{f}, \lambda_{p}, \mathrm{~cm}$ and they do not address signal-to-noise ratio versus $\mathrm{cm}$. They also do not use the low magnetic field approximation to simplify the calculations.

\section{The Resistance between the Flush Contacts on the Axis of Symmetry and the Common Mode Potential of the Partial Contacts off the Axis of Symmetry}

First we compute the resistance between the flush contact pair and the common mode potential of the partial contacts-both at zero magnetic field. To this end we map the rectangular plate in the $z$-plane of Figure 3(a) via a conformal 


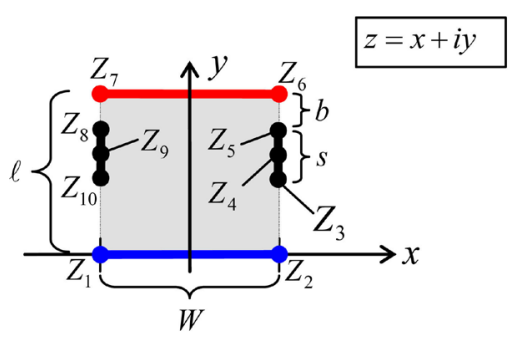

(a)

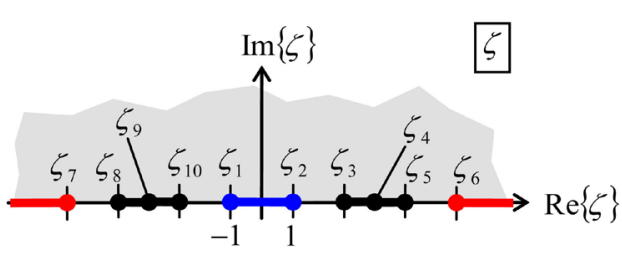

(b)

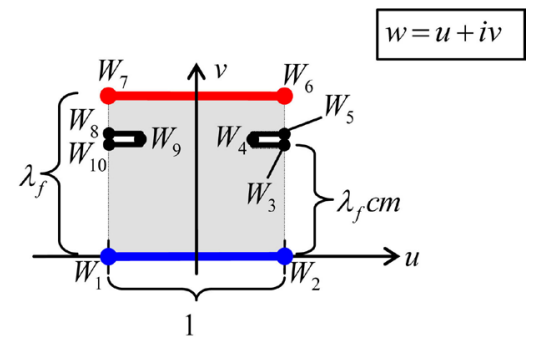

(c)

Figure 3. (a) Shows the original Hall plate in $z$-domain with mirror symmetry relative to the $y$-axis. (b) Upper half of the $\zeta$-plane with contacts on the real axis. (c) A rectangular plate where the partial contacts $\overline{W_{3} W_{4} W_{5}}$ and $\overline{W_{8} W_{9} W_{10}}$ are folded and parallel to the flush contacts $\overline{W_{1} W_{2}}$ and $\overline{W_{6} W_{7}}$. The aspect ratio $\overline{W_{2} W_{6}}: \overline{W_{1} W_{2}}$ is equal to the number of squares $\lambda_{f}$ between the flush contacts. If $1 \mathrm{~V}$ is applied to contact $\overline{W_{6} W_{7}}$ while $\overline{W_{1} W_{2}}$ is tied to ground potential the common mode potential of the partial contacts is equal to $\overline{W_{2} W_{3}}$. It holds $\overline{W_{1} W_{2}}=\overline{W_{6} W_{7}}$.

transformation onto the upper half of the zeta plane and from this into another rectangle in the $w$-plane. The partial contacts in the $w$-plane are parallel to lines of constant potential, which greatly facilitates the calculation of their potential and the calculation of the resistance between the other contacts.

A mapping from the $\zeta$-plane onto the $z$-plane is given by Schwartz-Christoffel's formula

$$
z=C_{1} \int_{0}^{\zeta} \frac{\mathrm{d} \zeta}{\sqrt{\zeta^{2}-1} \sqrt{\zeta^{2}-\zeta_{6}^{2}}} .
$$

Applying (6) to the width of the rectangle in the $z$-plane gives

$$
\frac{W}{2}=C_{1} \int_{0}^{1} \frac{\mathrm{d} \zeta}{\sqrt{\zeta^{2}-1} \sqrt{\zeta^{2}-\zeta_{6}^{2}}}=\frac{C_{1}}{\zeta_{6}} K\left(\frac{1}{\zeta_{6}}\right)
$$

The complete elliptic integral $K$ is defined in Appendix A. Applying (6) to the length of the rectangle in the $z$-plane gives

$$
\ell=C_{1} \int_{1}^{\zeta_{6}} \frac{\mathrm{d} \zeta}{\sqrt{\zeta^{2}-1} \sqrt{\zeta_{6}^{2}-\zeta^{2}}}=\frac{C_{1}}{\zeta_{6}} K^{\prime}\left(\frac{1}{\zeta_{6}}\right)
$$

$K^{\prime}$ is the complementary complete elliptic integral defined in Appendix A. Dividing (8) by (7) gives

$$
\frac{2 \ell}{W}=\frac{K^{\prime}\left(1 / \zeta_{6}\right)}{K\left(1 / \zeta_{6}\right)}
$$

Equation (9) defines $\zeta_{6}$. Applying (6) to $\overline{Z_{5} Z_{6}}$ and using (61) in [14] gives

$$
b=C_{1}\left(\int_{1}^{\zeta_{6}} \frac{\mathrm{d} \zeta}{\sqrt{\zeta^{2}-1} \sqrt{\zeta_{6}^{2}-\zeta^{2}}}-\int_{1}^{\zeta_{5}} \frac{\mathrm{d} \zeta}{\sqrt{\zeta^{2}-1} \sqrt{\zeta_{6}^{2}-\zeta^{2}}}\right)=\frac{C_{1}}{\zeta_{6}} F\left(\sqrt{\frac{\zeta_{6}^{2}-\zeta_{5}^{2}}{\zeta_{6}^{2}-1}}, \sqrt{1-\frac{1}{\zeta_{6}^{2}}}\right) .
$$

with the incomplete elliptic integral $F$ defined in Appendix A. (10) can be solved for $\zeta_{5}$ by use of the Jacobi-sn function (see Appendix A) 


$$
\zeta_{5}=\zeta_{6} \sqrt{1-\left(1-\frac{1}{\zeta_{6}^{2}}\right) \operatorname{sn}^{2}\left(\frac{b}{\ell} K^{\prime}\left(\frac{1}{\zeta_{6}}\right), \sqrt{1-\frac{1}{\zeta_{6}^{2}}}\right)} .
$$

In an analogous way one can apply (6) to $\overline{Z_{3} Z_{6}}$. This gives

$$
\zeta_{3}=\zeta_{6} \sqrt{1-\left(1-\frac{1}{\zeta_{6}^{2}}\right) \operatorname{sn}^{2}\left(\frac{b+s}{\ell} K^{\prime}\left(\frac{1}{\zeta_{6}}\right), \sqrt{1-\frac{1}{\zeta_{6}^{2}}}\right)} .
$$

From (12) one can compute $b+s$ from which one can subtract (10). With [21] it follows

$$
\frac{s}{\ell}=\frac{1}{K^{\prime}\left(\frac{1}{\zeta_{6}}\right)} F\left(\zeta_{6} \frac{\zeta_{5} \sqrt{\zeta_{5}^{2}-1} \sqrt{\zeta_{6}^{2}-\zeta_{3}^{2}}-\zeta_{3} \sqrt{\zeta_{3}^{2}-1} \sqrt{\zeta_{6}^{2}-\zeta_{5}^{2}}}{\zeta_{6}^{2}\left(\zeta_{3}^{2}+\zeta_{5}^{2}-1\right)-\zeta_{3}^{2} \zeta_{5}^{2}}, \sqrt{1-\frac{1}{\zeta_{6}^{2}}}\right) .
$$

From (11), (12), and [21] it follows

$$
\frac{2 b+s}{\ell}=\frac{1}{K^{\prime}\left(\frac{1}{\zeta_{6}}\right)} F\left(\zeta_{6} \frac{\zeta_{5} \sqrt{\zeta_{5}^{2}-1} \sqrt{\zeta_{6}^{2}-\zeta_{3}^{2}}+\zeta_{3} \sqrt{\zeta_{3}^{2}-1} \sqrt{\zeta_{6}^{2}-\zeta_{5}^{2}}}{\zeta_{6}^{2}\left(\zeta_{3}^{2}+\zeta_{5}^{2}-1\right)-\zeta_{3}^{2} \zeta_{5}^{2}}, \sqrt{1-\frac{1}{\zeta_{6}^{2}}}\right) .
$$

For the symmetric case $\mathrm{cm}=1 / 2$ it holds $2 b+s=\ell$. Then the first argument in the incomplete elliptic integral in (14) is equal to unity. This gives

$$
\zeta_{3} \zeta_{5}=\zeta_{6} \text { for } \mathrm{cm}=1 / 2 \text {. }
$$

By now we have expressed all parameters defining the contacts in the $\zeta$-plane by parameters of the $z$-plane. Turning to the mapping from the $\zeta$-plane onto the $w$-plane in Figure 3(c) we can again use the transformation of Schwartz-Christoffel

$$
w=C_{2} \int_{0}^{\zeta} \frac{\zeta^{2}-\zeta_{4}^{2}}{\sqrt{\zeta-1} \sqrt{\zeta-\zeta_{3}} \sqrt{\zeta-\zeta_{5}} \sqrt{\zeta-\zeta_{6}} \sqrt{\zeta+\zeta_{6}} \sqrt{\zeta+\zeta_{5}} \sqrt{\zeta+\zeta_{3}} \sqrt{\zeta+1}} \mathrm{~d} \zeta .
$$

Note that $\sqrt{\zeta-1} \sqrt{\zeta+1} \neq \sqrt{\zeta^{2}-1}$, because for $\zeta=-2$ we get $\sqrt{\zeta-1} \sqrt{\zeta+1} \rightarrow \sqrt{-3} \sqrt{-1}=i^{2} \sqrt{3}=-\sqrt{3}$ which is different from $\sqrt{\zeta^{2}-1} \rightarrow \sqrt{3}$. The integral (16) is more difficult than (6). It contains additional factors which are caused by the $90^{\circ}$ corners at points $W_{3}, W_{5}$ and by the $180^{\circ}$ turn at point $W_{4}$ (plus their symmetric counterparts at $W_{8}, W_{9}, W_{10}$ ).

The parameter $\zeta_{4}=-\zeta_{9}$ is defined by the requirement $\overline{W_{3} W_{4}}=\overline{W_{4} W_{5}}$ or $\overline{W_{3} W_{5}}=0$ which means that the contacts are folded in such a way that points $W_{3}$ and $W_{5}$ become identical. This gives

$$
0=\int_{\zeta_{3}}^{\zeta_{5}} \frac{\zeta^{2}-\zeta_{4}^{2}}{\sqrt{\zeta^{2}-\zeta_{3}^{2}} \sqrt{\zeta_{5}^{2}-\zeta^{2}}} \frac{\mathrm{d} \zeta}{\sqrt{\zeta^{2}-1} \sqrt{\zeta_{6}^{2}-\zeta^{2}}} .
$$

Equation (17) can be solved to give the parameter $\zeta_{4}$

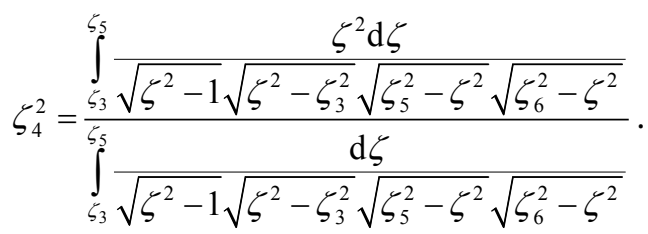


With the abbreviations (B4) defined in Appendix B Equation (18) becomes

$$
\zeta_{4}^{2}=G_{2} / G_{0} \text {. }
$$

The number of squares between the flush contacts is defined as the resistance between these contacts divided by the sheet resistance. With Figure $3(\mathrm{c})$ it is given by

$$
\lambda_{f}=\frac{\overline{W_{2} W_{6}}}{\overline{W_{1} W_{2}}}=\frac{\overline{W_{2} W_{3}}+\overline{W_{5} W_{6}}}{\overline{W_{1} W_{2}}}=\frac{\int_{1}^{\zeta_{3}}\left(\zeta_{4}^{2}-\zeta^{2}\right) g(\zeta) \mathrm{d} \zeta+\int_{\zeta_{5}}^{\zeta_{6}}\left(\zeta^{2}-\zeta_{4}^{2}\right) g(\zeta) \mathrm{d} \zeta}{2 \int_{0}^{1}\left(\zeta_{4}^{2}-\zeta^{2}\right) g(\zeta) \mathrm{d} \zeta} .
$$

For the common-mode potential of the partial contacts $\overline{W_{3} W_{4} W_{5}}$ at zero magnetic field we assume that contact $\overline{W_{6} W_{7}}$ is at potential $V_{i n}$ and contact $\overline{W_{1} W_{2}}$ is at zero potential, i.e. ground potential. From Figure 3 (c) we get

$$
\frac{1}{\mathrm{~cm}}=\frac{\overline{W_{2} W_{6}}}{\overline{W_{2} W_{3}}}=1+\frac{\overline{W_{5} W_{6}}}{\overline{W_{2} W_{3}}}=1+\frac{\int_{\zeta_{5}}^{\zeta_{6}}\left(\zeta^{2}-\zeta_{4}^{2}\right) g(\zeta) \mathrm{d} \zeta}{\int_{1}^{\zeta_{3}}\left(\zeta_{4}^{2}-\zeta^{2}\right) g(\zeta) \mathrm{d} \zeta}
$$

With the functions defined in Appendix B we may write (20), (21) like this

$$
\begin{gathered}
\frac{c m}{1-c m}=\frac{-\bar{G}_{2} G_{0}+\bar{G}_{0} G_{2}}{\overline{\bar{G}}_{2} G_{0}-\overline{\bar{G}}_{0} G_{2}}, \\
2 \lambda_{f} \mathrm{~cm}=\frac{\bar{G}_{2} G_{0}-\bar{G}_{0} G_{2}}{G_{2}^{\prime} G_{0}-G_{0}^{\prime} G_{2}} .
\end{gathered}
$$

For large impedance between the two contacts on the axis of mirror symmetry it follows from (9)

$$
\ell / W \rightarrow \infty \Leftrightarrow \zeta_{6} \rightarrow \infty \Leftrightarrow \lambda_{f} \rightarrow \infty \quad\left(\text { for } \zeta_{3}>1 \vee \zeta_{5}<\zeta_{6}\right)
$$

For $\zeta_{3} \rightarrow 1 \wedge \zeta_{5} \rightarrow \zeta_{6}$ all contacts touch and inputs and outputs are shorted.

\section{The Resistance between the Partial Contacts off the Axis of Symmetry}

We compute the resistance $\lambda_{p}$ between the partial contacts at zero magnetic field. For this purpose we map the interior of the rectangle in Figure 4 with both folded contacts on the single axis of mirror symmetry onto the upper half of the $\zeta$-plane in Figure 3(b).

$$
w=C_{5} \int_{0}^{\zeta} \frac{\zeta \mathrm{d} \zeta}{\sqrt{\zeta-1} \sqrt{\zeta-\zeta_{3}} \sqrt{\zeta-\zeta_{5}} \sqrt{\zeta-\zeta_{6}} \sqrt{\zeta+\zeta_{6}} \sqrt{\zeta+\zeta_{5}} \sqrt{\zeta+\zeta_{3}} \sqrt{\zeta+1}}
$$

The center of the folded bottom contact $\overline{W_{1} W_{2}}$ is mapped to the origin of the $\zeta$-plane, whereas the center of the folded top contact $\overline{W_{6} W_{7}}$ is mapped to infinity of the $\zeta$-plane. Therefore it does not show up in the integrand of the Schwartz-Christoffel integral (see also [22]). This leads to a simple expression for the number of squares between the partial contacts of the original device in Figure 3(a). 


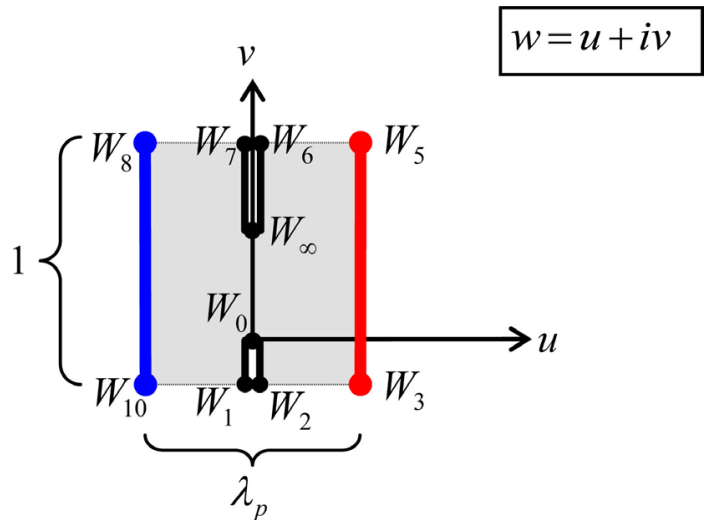

Figure 4. With conformal mappings (6) and (25) the original partial contacts in the $z$-plane of Figure 3(a) become flush contacts in the $w$-plane of this figure: $\overline{W_{8} W_{10}}$ and $\overline{W_{3} W_{5}}$. The original flush contacts in the $z$-plane of Figure $3(\mathrm{a})$ are folded in this figure: $\overline{W_{1} W_{2}}$ and $\overline{W_{6} W_{7}} \cdot \overline{W_{0} W_{2}} \neq \overline{W_{6} W_{\infty}}$ because at zero magnetic field different current flows through both contacts on the axis of symmetry along its way between left and right contacts (due to the asymmetry $c m \neq 1 / 2$ of the device).

$$
\lambda_{p}=2 \frac{\overline{W_{2} W_{3}}}{\overline{W_{3} W_{5}}}=2 \frac{\int_{\zeta_{3}}^{\zeta_{3}} \zeta g(\zeta) \mathrm{d} \zeta}{\int_{\zeta_{5}}^{\zeta_{5}} \zeta g(\zeta) \mathrm{d} \zeta}=\frac{2 \bar{G}_{1}}{G_{1}} .
$$

In (26) both integrals can be solved explicitly as functions of $\zeta_{3}, \zeta_{5}, \zeta_{6}$ (see Appendix B). Thus, in general the three parameters $\zeta_{3}, \zeta_{5}, \zeta_{6}$ are linked via $\lambda_{p}$

$$
L\left(\frac{2}{\lambda_{p}}\right)=\frac{\zeta_{6}^{2}-\zeta_{5}^{2}}{\zeta_{6}^{2}-\zeta_{3}^{2}} \frac{\zeta_{3}^{2}-1}{\zeta_{5}^{2}-1} .
$$

The modular lambda function $L$ is defined in Appendix A. The resistance between the partial contacts gets large for (see (13) or Figure 3(b))

$$
s / \ell \rightarrow 0 \Leftrightarrow \zeta_{3} \rightarrow \zeta_{5} \Leftrightarrow \lambda_{p} \rightarrow \infty \quad\left(\text { for } \zeta_{6}>1\right)
$$

Figure 4 shows that for $c m \neq 1 / 2$ the current through both original flush contacts is different, because the original partial contacts are closer to one than the other (see also Figure 3(a)). Therefore, $\overline{W_{0} W_{2}} \neq \overline{W_{6} W_{\infty}}$ and this gives $G_{1}^{\prime} \neq G_{1}^{\prime \prime}$. Only for $c m=1 / 2$ we have equal current over both flush contacts and this means $\overline{W_{0} W_{2}}=\overline{W_{6} W_{\infty}} \Leftrightarrow G_{1}^{\prime}=G_{1}^{\prime \prime}$ which means $\zeta_{3} \zeta_{5}=\zeta_{6}$ according to (B12). It holds

$$
\begin{aligned}
c m<1 / 2 & \Leftrightarrow G_{1}^{\prime}>G_{1}^{\prime \prime} \Leftrightarrow \zeta_{3} \zeta_{5}<\zeta_{6} \\
c m>1 / 2 & \Leftrightarrow G_{1}^{\prime}<G_{1}^{\prime \prime} \Leftrightarrow \zeta_{3} \zeta_{5}>\zeta_{6}
\end{aligned}
$$

Obviously the transformation $\mathrm{cm} \rightarrow 1-\mathrm{cm}$ keeps $\lambda_{f}, \lambda_{p}, G_{H 0}$ constant, while it swaps $G_{1}^{\prime}$ with $G_{1}^{\prime \prime}$. This corresponds to the transformation $\left(\zeta_{3}, \zeta_{5}, \zeta_{6}\right) \rightarrow\left(\zeta_{6} / \zeta_{5}, \zeta_{6} / \zeta_{3}, \zeta_{6}\right)$, which has the fix point $\zeta_{6}=\zeta_{3} \zeta_{5}$ for symmetric devices with $\mathrm{cm}=1 / 2$. 
For $c m=1 / 2$ it holds $Z_{4}=W / 2+i \ell / 2$ according to Figure $3($ a) \& Figure 3 (c). With (6) and with $\operatorname{sn}(K(k) / 2, k)=1 / \sqrt{1+\sqrt{1-k^{2}}}$ from [23] we get

$$
\zeta_{4}=\sqrt{\zeta_{6}} \text { for } c m=1 / 2 \text {. }
$$

Such a device can be mapped onto a disk with two perpendicular mirror symmetries like in Figure C2 in the appendix. Comparison of Figure C2(b) with Figure 3(b) gives relations between $\zeta_{3}, \zeta_{5}$ and the half aperture angles $\alpha_{1}, \alpha_{2}$ of the contacts. These angles are linked to the numbers of squares via $(\mathrm{C} 24, \mathrm{C} 25)$. Thus, the parameters $\zeta_{3}, \zeta_{5}$ are linked to the numbers of squares $\lambda_{f}, \lambda_{p}$ for $c m=1 / 2$ :

$$
\begin{gathered}
\lambda_{f}=\frac{K^{\prime}\left(\frac{\zeta_{3}+\zeta_{5}}{1+\zeta_{3} \zeta_{5}}\right)}{K\left(\frac{\zeta_{3}+\zeta_{5}}{1+\zeta_{3} \zeta_{5}}\right)} \\
\lambda_{p}=\frac{K^{\prime}\left(\frac{\zeta_{3}-\zeta_{5}}{\zeta_{3}+\zeta_{5}} \frac{1+\zeta_{3} \zeta_{5}}{1-\zeta_{3} \zeta_{5}}\right)}{K\left(\frac{\zeta_{3}-\zeta_{5}}{\zeta_{3}+\zeta_{5}} \frac{1+\zeta_{3} \zeta_{5}}{1-\zeta_{3} \zeta_{5}}\right)}
\end{gathered}
$$

Inverting (32), (33) gives $\zeta_{3}, \zeta_{5}$ as functions of $\lambda_{f}, \lambda_{p}$. With $L_{f}=L\left(\lambda_{f}\right)$, $L_{p}=L\left(\lambda_{p}\right)$ we get for $\mathrm{cm}=1 / 2$ :

$$
\begin{aligned}
& \zeta_{3}=\frac{1+L_{f} \sqrt{L_{p}}+\sqrt{\left(1-L_{f}\right)\left(1-L_{f} L_{p}\right)}}{\sqrt{L_{f}}\left(1+\sqrt{L_{p}}\right)} \\
& \zeta_{5}=\frac{1-L_{f} \sqrt{L_{p}}+\sqrt{\left(1-L_{f}\right)\left(1-L_{f} L_{p}\right)}}{\sqrt{L_{f}}\left(1-\sqrt{L_{p}}\right)}
\end{aligned}
$$

For $\mathrm{cm}=1 / 2 \wedge \lambda_{f}=\lambda_{p}$ it holds $\alpha_{1}=\alpha_{2}$ in Figure C2(a) in the appendix. Inserting this into (C15)-(C17) and into (C8)-(C10) gives

$$
\sqrt{\zeta_{6}}=\left(\sqrt{\zeta_{5}}+\sqrt{\zeta_{3}}\right) /\left(\sqrt{\zeta_{5}}-\sqrt{\zeta_{3}}\right)
$$

Inserting (15) into (36) gives

$$
\zeta_{6}=\zeta_{5} \zeta_{3}=\frac{1}{4}\left(1+\zeta_{3}+\sqrt{\zeta_{3}^{2}+6 \zeta_{3}+1}\right)^{2}
$$

which holds for devices with $90^{\circ}$ symmetry.

\section{The Hall Output Voltage at the Contacts on the Symmetry Axis}

Here we consider only the case when current flows between the original partial contacts off the symmetry axis and the output voltage is tapped between the original flush contacts on the axis of symmetry. At magnetic field the rectangle in Figure 4 is skewed by the Hall angle $\beta=\arctan \left(\mu_{H} B_{\perp}\right)$ [24] [25]. Then horizontal lines are at constant potential and $\operatorname{Im}\{w\}$ is the electric potential in 
Figure 5. The current streamlines are also straight and parallel to the left and right edges of the parallelogram in Figure 5. We use $m=\beta / \pi$ (which differs by a factor 2 from [12] and [24]).

A Schwartz-Christoffel mapping from the upper half-plane of Figure 3 onto the inside of the parallelogram in Figure 5 is

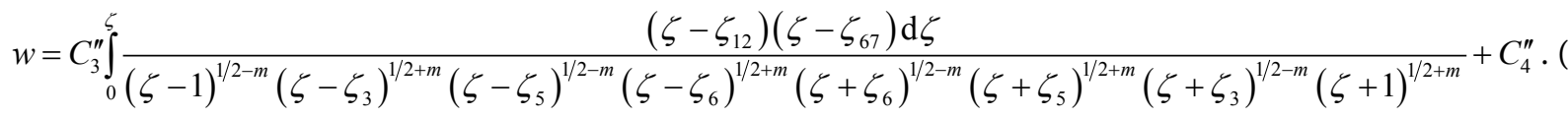

For small magnetic field it holds $|m| \ll 1$ and we may approximate $x^{m}=\exp (m \ln x) \cong 1+m \ln x . \zeta_{12}$ and $\zeta_{67}$ are determined by the requirements $\int_{-1}^{1} \cdots=0$ and $\int_{\zeta_{6}}^{\infty} \cdots+\int_{-\infty}^{-\zeta_{6}} \cdots=0$ with integrands like in (38). With the abbreviations in Appendix B this leads to the equations

$$
\begin{aligned}
& G_{2}^{\prime}+\zeta_{12} \zeta_{67} G_{0}^{\prime}-m H_{1}^{\prime}\left(\zeta_{12}+\zeta_{67}\right)=0 \\
& G_{2}^{\prime \prime}+\zeta_{12} \zeta_{67} G_{0}^{\prime \prime}-m H_{1}^{\prime \prime}\left(\zeta_{12}+\zeta_{67}\right)=0
\end{aligned}
$$

with the solutions

$$
\begin{aligned}
\zeta_{12} \zeta_{67} & =\frac{G_{2}^{\prime \prime} H_{1}^{\prime}-G_{2}^{\prime} H_{1}^{\prime \prime}}{G_{0}^{\prime} H_{1}^{\prime \prime}-G_{0}^{\prime \prime} H_{1}^{\prime}} \\
\zeta_{12}+\zeta_{67} & =\frac{1}{m} \frac{G_{0}^{\prime} G_{2}^{\prime \prime}-G_{0}^{\prime \prime} G_{2}^{\prime}}{G_{0}^{\prime} H_{1}^{\prime \prime}-G_{0}^{\prime \prime} H_{1}^{\prime}} .
\end{aligned}
$$

From (38) one gets

$$
\begin{aligned}
w_{3}-w_{5}= & C_{3}^{\prime \prime} \int_{\zeta=\zeta_{5}}^{\zeta_{3}} \frac{\left(\zeta-\zeta_{12}\right)\left(\zeta-\zeta_{67}\right)}{\sqrt{\zeta^{2}-1} \sqrt{\zeta^{2}-\zeta_{3}^{2}} \sqrt{\zeta^{2}-\zeta_{5}^{2}} \sqrt{\zeta^{2}-\zeta_{6}^{2}}}\{1+m[\ln (\zeta-1) \\
& -\ln (\zeta+1)+\ln \left(\zeta+\zeta_{3}\right)-\ln \left(\zeta-\zeta_{3}\right)+\ln \left(\zeta-\zeta_{5}\right) \\
& \left.\left.-\ln \left(\zeta+\zeta_{5}\right)+\ln \left(\zeta+\zeta_{6}\right)-\ln \left(\zeta-\zeta_{6}\right)\right]\right\} \mathrm{d} \zeta
\end{aligned}
$$

which is identical to

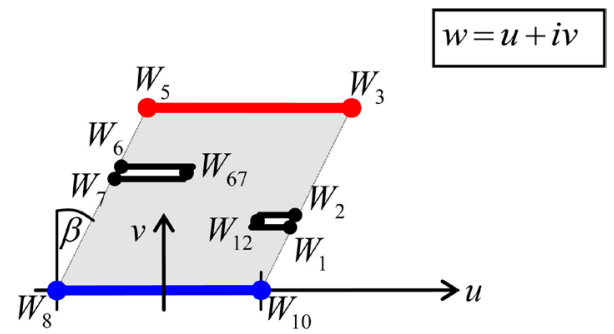

Figure 5. In the presence of a magnetic field the Hall plate of Figure 4 is mapped onto a rectangle which is skewed by the Hall angle $\beta$. The homogeneous current flows in streamlines parallel to $\overline{W_{3} W_{10}}$ between the original partial contacts at top and bottom of this figure. The equipotential lines are horizontal. Equipotential lines and current streamlines intersect at an angle of $\pi / 2-\beta$. The flush contacts of the original device in Figure 3(a) are folded in this figure and they are also horizontal. Thus, they do not distort the equipotential lines. The output voltage is $\left(\overline{W_{2} W_{3}}-\overline{W_{5} W_{6}}\right) \cos \beta$. The current is $\overline{W_{3} W_{5}} \cos ^{2} \beta / R_{\mathrm{sh}}$. 


$$
\begin{aligned}
& C_{3}^{\prime \prime} \int_{\zeta=\zeta_{5}}^{\zeta_{3}} \frac{\zeta^{2}-\left(\zeta_{12}+\zeta_{67}\right) \zeta+\zeta_{12} \zeta_{67}}{i^{2} \sqrt{\zeta^{2}-1} \sqrt{\zeta^{2}-\zeta_{3}^{2}} \sqrt{\zeta_{5}^{2}-\zeta^{2}} \sqrt{\zeta_{6}^{2}-\zeta^{2}}}\left\{1+m\left[\ln \left(\frac{\zeta-1}{\zeta+1} \frac{\zeta+\zeta_{3}}{\zeta-\zeta_{3}}\right)\right.\right. \\
& \left.\left.+i \pi+\ln \left(\zeta_{5}-\zeta\right)-\ln \left(\zeta+\zeta_{5}\right)+\ln \left(\zeta+\zeta_{6}\right)-i \pi-\ln \left(\zeta-\zeta_{6}\right)\right]\right\} \mathrm{d} \zeta \\
& =-C_{3}^{\prime \prime} \int_{\zeta=\zeta_{3}}^{\zeta_{5}} \frac{\zeta^{2}-\left(\zeta_{12}+\zeta_{67}\right) \zeta+\zeta_{12} \zeta_{67}}{i^{2}}(g(\zeta)+m h(\zeta)) \mathrm{d} \zeta
\end{aligned}
$$

The arbitrary scaling $w_{3}-w_{5}=1$ gives the real valued constant $C_{3}^{\prime \prime}$.

$$
C_{3}^{\prime \prime}=\frac{1}{G_{2}-\left(\zeta_{12}+\zeta_{67}\right) G_{1}+\zeta_{12} \zeta_{67} G_{0}+m\left(H_{2}-\left(\zeta_{12}+\zeta_{67}\right) H_{1}+\zeta_{12} \zeta_{67} H_{0}\right)}
$$

$C_{3}^{\prime \prime} \rightarrow 0$ for $m \rightarrow 0$ due to (42). Next we compute the potential at the sense contacts in Figure 5. With (38) we get

$$
\begin{aligned}
w_{1}-w_{10} & C_{3}^{\prime \prime} \int_{\zeta=-\zeta_{3}}^{-1} \frac{\left(\zeta-\zeta_{12}\right)\left(\zeta-\zeta_{67}\right)}{\sqrt{\zeta+\zeta_{6}} \sqrt{\zeta+\zeta_{5}} \sqrt{\zeta+\zeta_{3}} \sqrt{\zeta+1} \sqrt{\zeta-1} \sqrt{\zeta-\zeta_{3}} \sqrt{\zeta-\zeta_{5}} \sqrt{\zeta-\zeta_{6}}} \\
& \times\left\{1+m\left[\ln (\zeta-1)-\ln (\zeta+1)+\ln \left(\zeta+\zeta_{3}\right)-\ln \left(\zeta-\zeta_{3}\right)\right.\right. \\
& \left.\left.+\ln \left(\zeta-\zeta_{5}\right)-\ln \left(\zeta+\zeta_{5}\right)+\ln \left(\zeta+\zeta_{6}\right)-\ln \left(\zeta-\zeta_{6}\right)\right]\right\} \mathrm{d} \zeta \\
= & C_{3}^{\prime \prime} \int_{\zeta=-\zeta_{3}}^{-1} \frac{\zeta^{2}-\left(\zeta_{12}+\zeta_{67}\right) \zeta+\zeta_{12} \zeta_{67}}{\zeta+\zeta_{6} \sqrt{\zeta+\zeta_{5}} \sqrt{\zeta+\zeta_{3}} \sqrt{-\zeta-1} \sqrt{-\zeta+1} \sqrt{-\zeta+\zeta_{3}} \sqrt{-\zeta+\zeta_{5}} \sqrt{-\zeta+\zeta_{6}}} \\
& \times\left\{1+i m \pi(1-1-1+1-1)+m\left[\ln (-\zeta+1)-\ln (-\zeta-1)+\ln \left(\zeta+\zeta_{3}\right)\right.\right. \\
& \left.\left.-\ln \left(-\zeta+\zeta_{3}\right)+\ln \left(-\zeta+\zeta_{5}\right)-\ln \left(\zeta+\zeta_{5}\right)+\ln \left(\zeta+\zeta_{6}\right)-\ln \left(-\zeta+\zeta_{6}\right)\right]\right\} \mathrm{d} \zeta \\
= & C_{3}^{\prime \prime \prime} \int_{\zeta=1}^{\zeta_{3}} \frac{\zeta^{2}+\left(\zeta_{12}+\zeta_{67}\right) \zeta+\zeta_{12} \zeta_{67}}{i-\zeta+\zeta_{6} \sqrt{-\zeta+\zeta_{5}} \sqrt{-\zeta+\zeta_{3}} \sqrt{\zeta-1} \sqrt{\zeta+1} \sqrt{\zeta+\zeta_{3}} \sqrt{\zeta+\zeta_{5}} \sqrt{\zeta+\zeta_{6}}} \\
& \times\left\{1-i m \pi+m\left[\ln (\zeta+1)-\ln (\zeta-1)+\ln \left(-\zeta+\zeta_{3}\right)-\ln \left(\zeta+\zeta_{3}\right)\right.\right. \\
& \left.\left.+\ln \left(\zeta+\zeta_{5}\right)-\ln \left(-\zeta+\zeta_{5}\right)+\ln \left(-\zeta+\zeta_{6}\right)-\ln \left(\zeta+\zeta_{6}\right)\right]\right\} \mathrm{d} \zeta \\
= & C_{3}^{\prime \prime} \int_{\zeta=1}^{\zeta_{3}} \frac{\zeta^{2}+\left(\zeta_{12}+\zeta_{67}\right) \zeta+\zeta_{12} \zeta_{67}}{i}[(1-i m \pi) g(\zeta)-m h(\zeta)] \mathrm{d} \zeta \\
= & \frac{(-i-m \pi)\left(\bar{G}_{2}+\left(\zeta_{12}+\zeta_{67}\right) \bar{G}_{1}+\zeta_{12} \zeta_{67} \bar{G}_{0}\right)+i m\left(\bar{H}_{2}+\left(\zeta_{12}+\zeta_{67}\right) \bar{H}_{1}+\zeta_{12} \zeta_{67} \bar{H}_{0}\right)}{G_{2}-\left(\zeta_{12}+\zeta_{67}\right) G_{1}+\zeta_{12} \zeta_{67} G_{0}+m\left(H_{2}-\left(\zeta_{12}+\zeta_{67}\right) H_{1}+\zeta_{12} \zeta_{67} H_{0}\right)}
\end{aligned}
$$

Thus, the potential at this sense contact $\overline{12}$ is

$$
\begin{aligned}
& \operatorname{Im}\left\{w_{1}-w_{10}\right\} \\
& =\frac{-\left(\bar{G}_{2}+\left(\zeta_{12}+\zeta_{67}\right) \bar{G}_{1}+\zeta_{12} \zeta_{67} \bar{G}_{0}\right)+m\left(\bar{H}_{2}+\left(\zeta_{12}+\zeta_{67}\right) \bar{H}_{1}+\zeta_{12} \zeta_{67} \bar{H}_{0}\right)}{G_{2}-\left(\zeta_{12}+\zeta_{67}\right) G_{1}+\zeta_{12} \zeta_{67} G_{0}+m\left(H_{2}-\left(\zeta_{12}+\zeta_{67}\right) H_{1}+\zeta_{12} \zeta_{67} H_{0}\right)} \\
& =\frac{\lambda_{p}}{2}+\frac{2\left(\bar{G}_{2}-\left(\zeta_{12}+\zeta_{67}\right) m \bar{H}_{1}+\zeta_{12} \zeta_{67} \bar{G}_{0}\right)+\lambda_{p}\left(G_{2}-\left(\zeta_{12}+\zeta_{67}\right) m H_{1}+\zeta_{12} \zeta_{67} G_{0}\right)}{2 G_{1}\left(\zeta_{12}+\zeta_{67}\right) m} m+O(m)^{2}
\end{aligned}
$$

where we used (26) and neglected higher powers in $m$. At zero magnetic field the 
potential is $\lambda_{p} / 2$, which is half the supply voltage. Hence, the change in potential at sense contact $\overline{12}$ caused by small magnetic field is $d V_{\text {out }}=\operatorname{Im}\left\{w_{1}-w_{10}\right\}-\lambda_{p} / 2$. With $\mu_{H} d B_{\perp}=\tan \beta$ and (1) we define the low field Hall geometry factor for this single sense contact

$$
G_{H 0,12}=-\frac{\operatorname{Im}\left\{w_{1}-w_{10}\right\}-\lambda_{p} / 2}{R_{\mathrm{sh}} I_{\text {in }} \tan \beta} .
$$

In (48) the negative sign in front of the fraction is needed to make the Hall geometry factor positive, even though the potential at this contact $\overline{12}$ decreases with rising field according to Figure 5.

The Hall supply current is equal to the integral of the current density component perpendicular to the contact along the contact: $I_{\text {in }}=t_{H} \int_{W_{5}}^{W_{3}} \mathrm{~d} J_{v}=t_{H} J\left(w_{3}-w_{5}\right) \cos \beta$ with $J$ being the homogeneous current density. Computing the scalar product of both sides of general Ohm's law $\boldsymbol{J}=\sigma_{0} \boldsymbol{E}-\mu_{H} \boldsymbol{J} \times \boldsymbol{B}$ with $\boldsymbol{J}$ we get $J=\sigma_{0} E \cos \beta \quad(\boldsymbol{J}, \boldsymbol{E}, \boldsymbol{B}$ are the vector fields of current density, electric field and magnetic flux density with $J=|\boldsymbol{J}|$ and $E=|\boldsymbol{E}|$ ). Finally, $E=1$ in Figure 5, because the voltage drop over the device is $\operatorname{Im}\left\{W_{3}\right\}$ and the distance between both supply contacts is also $\operatorname{Im}\left\{W_{3}\right\}$ and the electric field is homogeneous. This gives

$$
I_{\text {in }}=\left(w_{3}-w_{5}\right) \sigma_{0} t_{H} \cos ^{2} \beta
$$

with $w_{3}-w_{5}=1$ from (45) [26]. With (B23), (B24), and (B41) in Appendix B we get

$$
G_{H 0,12}=\frac{1}{\pi G_{1}}\left\{-\frac{G_{0}^{\prime} \bar{G}_{2}-G_{2}^{\prime} \bar{G}_{0}}{G_{0}^{\prime} G_{2}-G_{2}^{\prime} G_{0}} H_{1}+\bar{H}_{1}-2 \lambda_{f} c m H_{1}^{\prime}\right\} .
$$

For the other sense contact $\overline{67}$ we get

$$
=C_{3}^{\prime \prime} \int_{\zeta=-\zeta_{5}}^{-\zeta_{6}} \frac{\left(\zeta-\zeta_{12}\right)\left(\zeta-\zeta_{67}\right)}{\sqrt{\zeta+\zeta_{6}} \sqrt{\zeta+\zeta_{5}} \sqrt{\zeta+\zeta_{3}} \sqrt{\zeta+1} \sqrt{\zeta-1} \sqrt{\zeta-\zeta_{3}} \sqrt{\zeta-\zeta_{5}} \sqrt{\zeta-\zeta_{6}}}
$$$$
\times\left\{1+m\left[\ln (\zeta-1)-\ln (\zeta+1)+\ln \left(\zeta+\zeta_{3}\right)-\ln \left(\zeta-\zeta_{3}\right)\right.\right.
$$$$
\left.\left.+\ln \left(\zeta-\zeta_{5}\right)-\ln \left(\zeta+\zeta_{5}\right)+\ln \left(\zeta+\zeta_{6}\right)-\ln \left(\zeta-\zeta_{6}\right)\right]\right\} \mathrm{d} \zeta
$$$$
=-C_{3}^{\prime \prime} \int_{\zeta=\zeta_{5}}^{\zeta_{6}} \frac{\zeta^{2}+\left(\zeta_{12}+\zeta_{67}\right) \zeta+\zeta_{12} \zeta_{67}}{i^{7}}\{g(\zeta)[1+i m \pi(1-1+1-1+1-1-1)]-m h(\zeta)\} \mathrm{d} \zeta
$$$$
=\frac{\left(\overline{\bar{G}}_{2}+\left(\zeta_{12}+\zeta_{67}\right) \overline{\bar{G}}_{1}+\zeta_{12} \zeta_{67} \overline{\bar{G}}_{0}\right)(-i-m \pi)+i m\left(\overline{\bar{H}}_{2}+\left(\zeta_{12}+\zeta_{67}\right) \overline{\bar{H}}_{1}+\zeta_{12} \zeta_{67} \overline{\bar{H}}_{0}\right)}{G_{2}-\left(\zeta_{12}+\zeta_{67}\right) G_{1}+\zeta_{12} \zeta_{67} G_{0}+m\left(H_{2}-\left(\zeta_{12}+\zeta_{67}\right) H_{1}+\zeta_{12} \zeta_{67} H_{0}\right)}
$$

$$
\begin{aligned}
& \operatorname{Im}\left\{w_{7}-w_{8}\right\} \\
& =\frac{\lambda_{p}}{2}+\frac{2\left(\overline{\bar{G}}_{2}-\left(\zeta_{12}+\zeta_{67}\right) m \overline{\bar{H}}_{1}+\zeta_{12} \zeta_{67} \overline{\bar{G}}_{0}\right)+\lambda_{p}\left(G_{2}-\left(\zeta_{12}+\zeta_{67}\right) m H_{1}+\zeta_{12} \zeta_{67} G_{0}\right)}{2 G_{1}\left(\zeta_{12}+\zeta_{67}\right) m} m+O(m)^{2}
\end{aligned}
$$

We define 


$$
G_{H 0,67}=\frac{\operatorname{Im}\left\{w_{7}-w_{8}\right\}-\lambda_{p} / 2}{R_{\mathrm{sh}} I_{\text {in }} \tan \beta},
$$

which gives

$$
G_{H 0,67}=\frac{1}{\pi G_{1}}\left\{\frac{G_{0}^{\prime} \overline{\bar{G}}_{2}-G_{2}^{\prime} \overline{\bar{G}}_{0}}{G_{0}^{\prime} G_{2}-G_{2}^{\prime} G_{0}} H_{1}-\overline{\bar{H}}_{1}-2 \lambda_{f}(1-c m) H_{1}^{\prime}\right\} .
$$

Obviously, (50) and (54) are different: The magnetic sensitivity of the Hall signal at the two contacts on the axis of mirror symmetry is different. For $\mathrm{cm}>1 / 2$ more current is shunted via contact $\overline{67}$ than via $\overline{12}$, and therefore $G_{H 0,12}>G_{H 0,67}$. Thus, the output contact that is closer to a supply contact has smaller Hall signal. It also follows that contacts on the axis of mirror symmetry have a common mode of $1 / 2$ at zero magnetic field, but their common mode changes when magnetic field is applied. It depends on the sign of the magnetic field if the common mode increases or decreases.

The total Hall geometry factor is the sum of Hall geometry factors of both output contacts

$$
\begin{aligned}
G_{H 0} & =G_{H 0,12}+G_{H 0,67} \\
& =\frac{1}{\pi G_{1}}\left\{\frac{G_{0}^{\prime}\left(\overline{\bar{G}}_{2}-\bar{G}_{2}\right)-G_{2}^{\prime}\left(\overline{\bar{G}}_{0}-\bar{G}_{0}\right)}{G_{0}^{\prime} G_{2}-G_{2}^{\prime} G_{0}} H_{1}+\bar{H}_{1}-\overline{\bar{H}}_{1}-2 \lambda_{f} H_{1}^{\prime}\right\}
\end{aligned}
$$

With (B23), (B24), and (B41) in Appendix B we finally get

$$
G_{H 0}=\frac{1}{\pi G_{1}}\left\{\bar{H}_{1}-\overline{\bar{H}}_{1}+\frac{\left(\overline{\bar{G}}_{2}-\bar{G}_{2}\right)\left(G_{0}^{\prime \prime} H_{1}^{\prime}-G_{0}^{\prime} H_{1}^{\prime \prime}\right)-\left(\overline{\bar{G}}_{0}-\bar{G}_{0}\right)\left(G_{2}^{\prime \prime} H_{1}^{\prime}-G_{2}^{\prime} H_{1}^{\prime \prime}\right)}{G_{0}^{\prime \prime} G_{2}^{\prime}-G_{0}^{\prime} G_{2}^{\prime \prime}}\right\}
$$

For $\mathrm{cm}=1 / 2$ it holds $H_{1}^{\prime}=-H_{1}^{\prime \prime}$. This is readily proved by a transformation of the integration variable $\zeta=\zeta_{3} \zeta_{5} / \zeta^{\prime}$ in (B35) and by (15). With (B41) it follows $H_{1}=0$. Moreover, (50) and (54) must be equal due to the symmetry. Thus, $\bar{H}_{1}=-\overline{\bar{H}}_{1}$. Finally

$$
G_{H 0}=\frac{2}{\pi G_{1}}\left\{\bar{H}_{1}-\lambda_{f} H_{1}^{\prime}\right\} \text { for } c m=1 / 2
$$

(57), (34), and (35) give the weak field Hall geometry factor of devices with $\mathrm{cm}=1 / 2$, i.e. with two perpendicular mirror symmetries, as function of the numbers of squares. An alternative formula for this Hall geometry factor was given in (5a-c) in [1]. Although there is no apparent similarity in the two formulae their numerical results are identical.

\section{Discussion of the Hall Geometry Factor}

The Hall geometry factor $G_{H 0}$, the common mode $\mathrm{cm}$, and the numbers of squares $\lambda_{f}, \lambda_{p}$ at low magnetic field are functions of three parameters $\zeta_{3}, \zeta_{5}, \zeta_{6}$ which are subject to the relations $1 \leq \zeta_{3} \leq \zeta_{5} \leq \zeta_{6}$. On the other hand $G_{H 0}$ is an implicit function of the three parameters $\lambda_{f}, \lambda_{p}, \mathrm{~cm}$, which we study 
in the following. Thereby the quantity $G_{H 0} / \sqrt{\lambda_{f} \lambda_{p}}$ plays an important role. This ratio of Hall geometry factor over the square-root of the product of numbers of squares of inputs and outputs sums up the effect of the shape of the device on the signal-to-noise ratio at a fixed power dissipation (SNRP - P denotes fixed power). This is explained in [1] and there we also saw that $G_{H 0} / \sqrt{\lambda_{f} \lambda_{p}}$ cannot exceed an upper limit of $\sqrt{2} / 3 \cong 0.471$, which corresponds to Hall plates of highest symmetry (i.e. $\lambda_{f}=\lambda_{p}$ and $\mathrm{cm}=1 / 2$ ) with mid-sized contacts having $\lambda_{f}=\lambda_{p}=\sqrt{2}$ (see Figure 3 in [1]). If the symmetry is reduced by having different input and output resistances $\lambda_{f} \neq \lambda_{p}$ but still $\mathrm{cm}=1 / 2$ this will give notably smaller SNRP (see Figure 3 in [1]). The main motivation for this work is to answer the question whether a reduction in symmetry via $\mathrm{cm} \neq 1 / 2$ leads to an equally pronounced drop in SNRP or to just a minor drop. With the results of the preceding sections we can answer this and we also know the contact size (i.e. $\lambda_{f}$ and $\lambda_{p}$ ) for the maximum SNRP for every $\mathrm{cm}$. With these findings one can optimize Vertical Hall effect devices of the type in Figure 1(c), as shown in Appendix C.

\section{The Reduction in SNR of Hall Plates with Four Contacts and $\mathrm{cm} \neq$ $1 / 2$}

Here we are looking for the maximum of $G_{H 0} / \sqrt{\lambda_{f} \lambda_{p}}$ for all allowed values of the three parameters $\zeta_{3}, \zeta_{5}, \zeta_{6}$ under the additional constraint that $\mathrm{cm}$ has a fixed value between zero and one. To this end we use (22), (23), (26) and (56). However, the numerical evaluation of the involved integrals is tricky and therefore we need to use some identities and transformations of Appendix B. For the optimization algorithm it is also important to deal with the allowed range of parameters $1 \leq \zeta_{3} \leq \zeta_{5} \leq \zeta_{6}$. If the algorithm leaves this allowed range during its search, $G_{H 0} / \sqrt{\lambda_{f} \lambda_{p}}$ will get complex valued and the algorithm will have troubles to find the maximum. We avoid this problem by introducing additional parameters $x, y, z$ via $\zeta_{3}=1+x^{2}, \quad \zeta_{5}=1+x^{2}+y^{2}, \quad \zeta_{6}=1+x^{2}+y^{2}+z^{2}$. The new parameters $x, y, z$ can attain any real values without leaving the allowed region of $1<\zeta_{3}<\zeta_{5}<\zeta_{6}$. In spite of all these provisions the search for maximum $G_{H 0} / \sqrt{\lambda_{f} \lambda_{p}}$ lasted several hours for $\mathrm{cm} \rightarrow 0$ or $\mathrm{cm} \rightarrow 1$ (for details of the computer used see section 6).

In Figure 6 and Table 1 we chose specific values for $\mathrm{cm}$ and varied $\lambda_{f}$ and $\lambda_{p}$ to maximize $G_{H 0} / \sqrt{\lambda_{f} \lambda_{p}}$. This maximum $G_{H 0, \text { opt }} / \sqrt{\lambda_{f, o p t} \lambda_{p, o p t}}$ is located in $\lambda_{f}=\lambda_{f, \text { opt }}, \lambda_{p}=\lambda_{p, \text { opt }}$. The plot in Figure 6(a) shows that $G_{H 0, \text { opt }} / \sqrt{\lambda_{f, \text { opt }} \lambda_{p, o p t}}$ has a flat maximum versus $\mathrm{cm}$ near $\mathrm{cm}=0.5$ : in $|\mathrm{cm}-0.5|<0.1$ we loose less than $2.1 \%$ in $G_{H 0} / \sqrt{\lambda_{f} \lambda_{p}}$ w.r.t. its maximum, in $|c m-0.5|<0.2$ we loose less than $8.7 \%$ and in $|c m-0.5|<0.3$ we lose less than $20.8 \%$. Thereby, in all these common mode ranges the quantity $G_{H 0} / \sqrt{\lambda_{f} \lambda_{p}}$ evaluated at $\lambda_{f}=\lambda_{p}=\sqrt{2}$ is nearly as large as the maximum $G_{H 0, \text { opt }} / \sqrt{\lambda_{f, o p t} \lambda_{p, o p t}}$. The Hall geometry factor $G_{H 0, \text { opt }}=G_{H 0}\left(\lambda_{f}=\lambda_{f, \text { opt }}, \lambda_{p}=\lambda_{f, \text { opt }}\right)$ is similar to $G_{H 0}\left(\lambda_{f}=\lambda_{p}=\sqrt{2}\right)$ except for $c m<0.05$ or $c m>0.95$ (see Figure 6(b)). 
Figure $6(\mathrm{c})$ shows the exact location $\lambda_{f, o p t}, \lambda_{p, o p t}$ of the maximum $G_{H 0, \text { opt }} / \sqrt{\lambda_{f, o p t} \lambda_{p, o p t}}$ at various $\mathrm{cm}: \lambda_{p, \text { opt }}$ remains closely below $\sqrt{2}$ for all common modes, but $\lambda_{f, o p t}$ increases drastically for $\mathrm{cm} \rightarrow 0$ or $\mathrm{cm} \rightarrow 1$ (note that $\lambda_{f} R_{\mathrm{sh}}$ is the resistance between the two contacts on the axis of mirror symmetry). Interestingly, at extreme common modes $G_{H 0, o p t}$ remains larger than 0.321, whereas $G_{H 0}\left(\lambda_{f}=\lambda_{p}=\sqrt{2}\right)$ decreases to zero (see Figure 6(b)). Figure 6(d) shows how $\zeta_{3, \text { opt }} / \zeta_{5, \text { opt }}$ and $\zeta_{5, \text { opt }} / \zeta_{6, \text { opt }}$ vary versus $\mathrm{cm} \cdot \zeta_{6, \text { opt }}$ has a minimum in $c m=1 / 2$, whereas it increases drastically at extreme common modes (not shown).

For $c m=1 / 2 \wedge \lambda_{f}=\lambda_{p}=\sqrt{2}$ the SNRP is maximal. The exact location of this global maximum follows from (27) and (37).

$$
\begin{gathered}
\zeta_{3, \text { max }}=-1+2 \sqrt{2}+2 \sqrt{2-\sqrt{2}} \cong 3.35916 \\
\zeta_{5, \text { max }}=1+2 \sqrt{2}+2 \sqrt{2+\sqrt{2}} \cong 7.52395 \\
\zeta_{6, \text { max }}=7+4 \sqrt{2}+2 \sqrt{20+14 \sqrt{2}} \cong 25.27410
\end{gathered}
$$

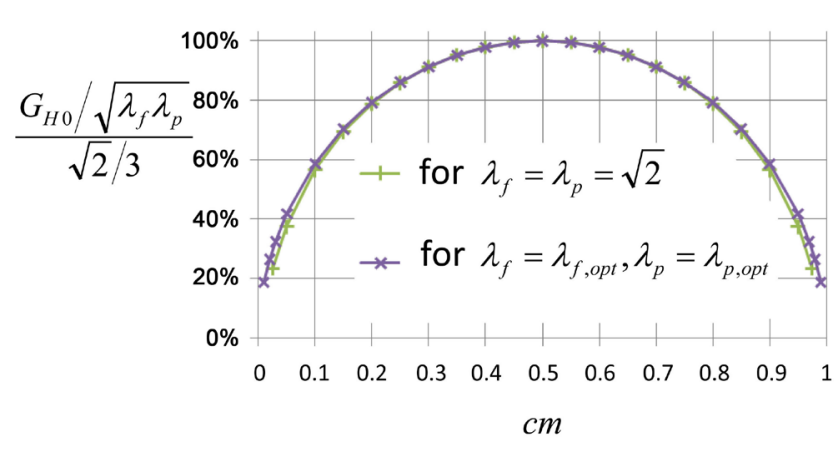

(a)

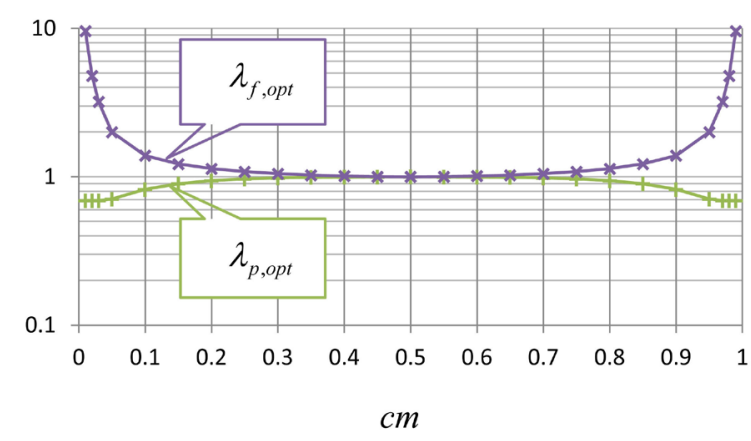

(c)

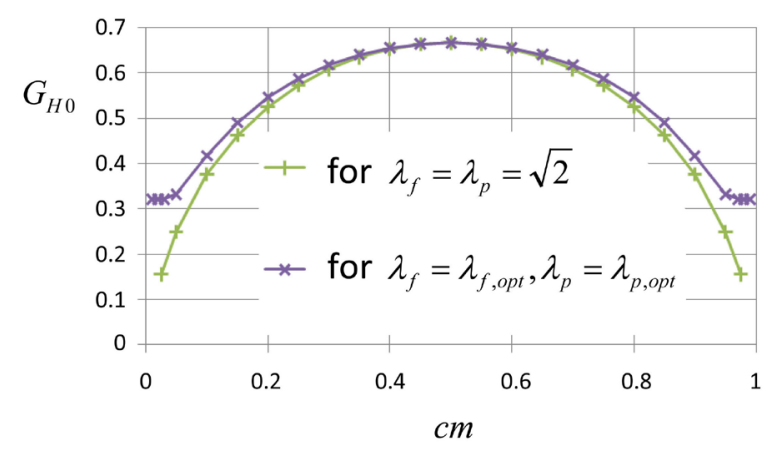

(b)

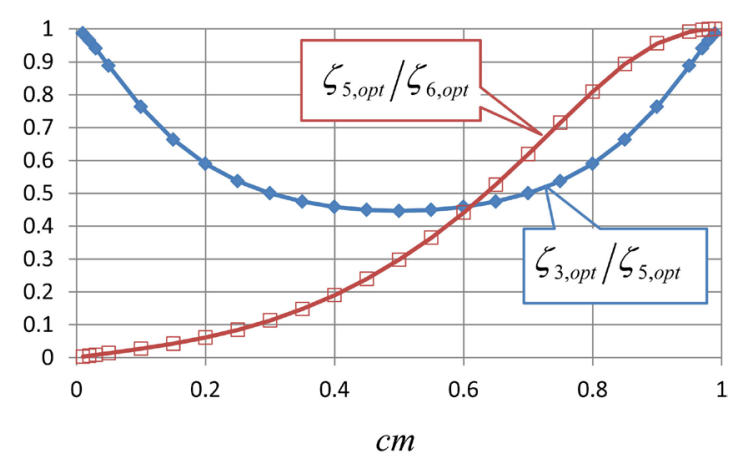

(d)

Figure 6. (a) Maximum of $3 G_{H 0} / \sqrt{2 \lambda_{f} \lambda_{p}}$ versus $\lambda_{f}, \lambda_{p}$ for varying common mode $\mathrm{cm}$. The maximum possible value of $3 G_{H 0} / \sqrt{2 \lambda_{f} \lambda_{p}}$ at $c m=1 / 2$ is 1 , and for $c m \neq 1 / 2$ it is smaller. In $\lambda_{f}=\lambda_{p}=\sqrt{2}$ the parameter $3 G_{H 0} / \sqrt{2 \lambda_{f} \lambda_{p}}$ is only a tiny bit smaller than in $\lambda_{f}=\lambda_{f, o p t}, \lambda_{p}=\lambda_{p, o p t}$. (b) The Hall geometry factor $G_{H 0}$ in $\lambda_{f}=\lambda_{f, o p t}, \lambda_{p}=\lambda_{p, o p t}$ and in $\lambda_{f}=\lambda_{p}=\sqrt{2}$ : they are similar in the range $0.3<\mathrm{cm}<0.7$ but they are quite different for $\mathrm{cm} \leq 0.05$ or $\mathrm{cm} \geq 0.95$. (c) The number of squares $\lambda_{f, \text { opt }}, \lambda_{p, \text { opt }}$ are close to $\sqrt{2}$ in the range $0.3<\mathrm{cm}<0.7$ but outside this range $\lambda_{f, \text { opt }}$ increases sharply while $\lambda_{p, o p t}$ decreases moderately. (d) Variation of $\zeta_{3, \text { opt }} / \zeta_{5, \text { opt }}$ and $\zeta_{5, \text { opt }} / \zeta_{6, \text { opt }}$ for varying common mode $\mathrm{cm}$. 
On the other hand, it is known from [27] that such devices of highest symmetry have

$$
G_{H 0}\left(\zeta_{3, \max }, \zeta_{5, \max }, \zeta_{6, \max }\right)=2 / 3 .
$$

A strict mathematical proof of (61) based on (57) seems to be challenging and has not yet been accomplished.

\section{Numerical Verification}

Let us consider a first rectangular Hall plate of Figure 3(a) with $t_{\mathrm{H}}=W=1 \mathrm{~m}, \ell$ $=1.770130 \mathrm{~m}, b=0.162917 \mathrm{~m}, s=0.239699 \mathrm{~m}$. This gives a common mode $\mathrm{cm}=$ 0.85 with maximum $G_{H 0} / \sqrt{\lambda_{f} \lambda_{p}}$ and $\lambda_{f} \cong 1.726, \lambda_{p} \cong 1.270$ (see italic line in Table 1). A second rectangular Hall plate with $t_{\mathrm{H}}=W=1 \mathrm{~m}, \ell=1.435180 \mathrm{~m}, b=$ $0.099209 \mathrm{~m}, s=0.113905 \mathrm{~m}$ also has a common mode $\mathrm{cm}=0.85$, yet with $\lambda_{f}=\lambda_{p} \cong 1.414$ and with slightly smaller $G_{H 0} / \sqrt{\lambda_{f} \lambda_{p}} \quad$ (see italic line in Table 2). Table 3 compares the parameters of both Hall plates obtained by our theory with results from finite element simulations (FEM) done with COMSOL MULTIPHYSICS. The FEM simulation used a 2D static conduction model with a conductivity tensor with values $1 /\left(1+0.01^{2}\right)$ in the main diagonal and $\pm 0.01 /(1+$ $\left.0.01^{2}\right)$ in the minor diagonal. This corresponds to a small Hall angle of $0.573^{\circ}$. The triangular meshing was fine: it had more than 3.6 million elements. Lagrange multiplier was used to get accurate values of the current through the supply contacts. The values of the analytical theory were obtained with MATHEMATICA 8.0 (see also details in Appendix B.3). All computations were carried out on a desktop computer with Intel Core i7-4930K CPU, ASRock X79 Extreme 6 mainboard, PNY Quadro K2000D graphics board with $64 \mathrm{~GB}$ RAM and $3.4 \mathrm{GHz}$ clock cycle.

The differences between the analytical theory and the FEM simulation are smaller than $0.03 \%$ for all values of Table 3, except for $G_{H 0,12}$ and $G_{H 0,67}$. There the difference is up to $0.1 \%$, probably due to insufficient meshing. The difference in the noise relevant parameter $G_{H 0} / \sqrt{\lambda_{f} \lambda_{p}}$ between both Hall plates is only $1.4 \%$, and it is correctly given by the theory. The difference in Hall geometry factor between both Hall plates is $5.8 \%$, and it is also given accurately by the theory. Also the ratios of contributions to magnetic sensitivity of contact $\overline{12}$ versus contact $\overline{67}$ are predicted accurately by the theory: it is 2.69 for the first Hall plate and 3.17 for the second Hall plate. The numbers of squares $\lambda_{f}, \lambda_{p}$ of the first Hall plate differ by a factor of about 1.36, whereas they are identical for the second Hall plate - all are given precisely by the theory.

From a practical view point this example shows that a Hall plate with a fairly pronounced common mode of 0.85 may still achieve a good noise performance. It has the largest SNRP if input and output resistances differ by $36 \%$. Then its SNRP is only $30 \%$ less than for an optimum Hall plate with $90^{\circ}$ symmetry and 1.4 squares impedance. If the electronic circuit requires equal input and output resistances at the same common mode of 0.85 , the SNRP loss is only slightly larger ( $31 \%$ instead of $30 \%$ for $\left.\lambda_{f}=\lambda_{p}=\sqrt{2}\right)$. 


\section{Summary}

This work gave an analytical theory on the signal-to-thermal-noise ratio (SNR) of Hall plates with reduced symmetry, where the common mode potential of the two output contacts was not midway between the two supply potentials. The method

Table 1. $G_{H 0, o p t} / \sqrt{\lambda_{f, o p t} \lambda_{p, o p t}}$ for noise-optimized Hall plates with reduced symmetry $c m \neq 1 / 2$. Each line of the table gives the global maximum of $G_{H 0} / \sqrt{\lambda_{f} \lambda_{p}}$ if the common mode is fixed to the specific value of the leftmost column while $\lambda_{f}, \lambda_{p}$ are varied. This maximum is obtained for $\lambda_{f}=\lambda_{f, \text { opt }}, \lambda_{p}=\lambda_{p, \text { opt }}$. It is irrelevant if $\lambda_{f}$ or $\lambda_{p}$ refer to the input, however, $\lambda_{f}$ refers to those two contacts, which lie on the single axis of mirror symmetry. $G_{H 0, o p t}$ is the Hall geometry factor at $\lambda_{f}=\lambda_{f, \text { opt }}, \lambda_{p}=\lambda_{p, o p t}$, and $\zeta_{3, \text { opt }}, \zeta_{5, \text { opt }}, \zeta_{6, \text { opt }}$ are the corresponding $\zeta$-parameters of (9), (11), (12). The data of this table is plotted in graphical form in Figures 6(a)-(d). SNRP loss is the reduction in $G_{H 0, \text { opt }} / \sqrt{\lambda_{f, \text { opt }} \lambda_{p, o p t}}$ compared to the overall maximum at $\mathrm{cm}=1 / 2$.

\begin{tabular}{|c|c|c|c|c|c|c|c|c|}
\hline $\mathrm{cm}$ & SNRP loss & $\max \frac{G_{H 0}}{\sqrt{\lambda_{f} \lambda_{p}}}$ & $\lambda_{f, o p t}$ & $\lambda_{p, o p t}$ & $G_{H 0, o p t}$ & $\zeta_{3, o p t}$ & $\zeta_{5, o p t}$ & $\zeta_{6, o p t}$ \\
\hline 0.01 & $-81.3 \%$ & 0.08820403 & 13.5684191 & 0.97714897 & 0.3211689 & 1.0126975 & 1.4154623 & $9.316 \mathrm{E}+17$ \\
\hline 0.02 & $-73.5 \%$ & 0.12473934 & 6.78427221 & 0.97715402 & 0.3211712 & 1.0126981 & 1.4154656 & 51666217 \\
\hline 0.03 & $-67.6 \%$ & 0.15277365 & 4.52444172 & 0.97734957 & 0.3212595 & 1.0127186 & 1.4155907 & 426524.66 \\
\hline 0.05 & $-58.2 \%$ & 0.19716117 & 2.83246755 & 1.00077903 & 0.33195 & 1.0153938 & 1.4313833 & 2094.4638 \\
\hline 0.10 & $-41.4 \%$ & 0.27614336 & 1.96616646 & 1.16343939 & 0.4176540 & 1.0549779 & 1.6190136 & 137.64742 \\
\hline 0.15 & $-29.7 \%$ & 0.33163219 & 1.72558712 & 1.27010636 & 0.4909590 & 1.1338450 & 1.9121322 & 65.026289 \\
\hline 0.20 & $-20.8 \%$ & 0.37346478 & 1.60523057 & 1.33229454 & 0.5461582 & 1.2528145 & 2.2935627 & 44.890437 \\
\hline 0.25 & $-13.9 \%$ & 0.40569653 & 1.53222002 & 1.36884971 & 0.5875431 & 1.4165467 & 2.771975 & 35.952151 \\
\hline 0.30 & $-8.7 \%$ & 0.43040045 & 1.48401527 & 1.3903129 & 0.6182273 & 1.6326833 & 3.3638489 & 31.098303 \\
\hline 0.35 & $-4.8 \%$ & 0.44875400 & 1.45144223 & 1.4027452 & 0.6403208 & 1.9129024 & 4.0933519 & 28.218344 \\
\hline 0.40 & $-2.1 \%$ & 0.46146003 & 1.43019915 & 1.4096867 & 0.6552298 & 2.2746276 & 4.9945976 & 26.49560 \\
\hline 0.45 & $-0.5 \%$ & 0.46893594 & 1.41813302 & 1.41316212 & 0.6638470 & 2.7437445 & 6.1154838 & 25.567890 \\
\hline 0.50 & $0.0 \%$ & 0.47140451 & 1.41384147 & 1.41435319 & 0.6666119 & 3.3591589 & 7.5239390 & 25.274102 \\
\hline 0.55 & $-0.5 \%$ & 0.46893594 & 1.41813133 & 1.41316432 & 0.6638471 & 4.1808368 & 9.3185630 & 25.567722 \\
\hline 0.60 & $-2.1 \%$ & 0.46146003 & 1.4301982 & 1.40968893 & 0.6552301 & 5.3048460 & 11.648275 & 26.495482 \\
\hline 0.65 & $-4.8 \%$ & 0.44875400 & 1.45144239 & 1.40274751 & 0.6403214 & 6.8937092 & 14.751561 & 28.218323 \\
\hline 0.70 & $-8.7 \%$ & 0.43040045 & 1.48401705 & 1.39031541 & 0.6182282 & 9.2449019 & 19.047404 & 31.098440 \\
\hline 0.75 & $-13.9 \%$ & 0.40569653 & 1.53222002 & 1.36884971 & 0.5875431 & 12.969869 & 25.380138 & 35.952151 \\
\hline 0.80 & $-20.8 \%$ & 0.37346478 & 1.60523879 & 1.33229834 & 0.546160 & 19.572788 & 35.832430 & 44.891549 \\
\hline 0.85 & $-29.7 \%$ & 0.33163219 & 1.72558712 & 1.27010636 & 0.4909589 & 34.007214 & 57.350248 & 65.026289 \\
\hline 0.90 & $-41.4 \%$ & 0.27614336 & 1.96616645 & 1.16343939 & 0.4176540 & 85.019310 & 130.47422 & 137.64742 \\
\hline 0.95 & $-58.2 \%$ & 0.19716117 & 2.83241167 & 1.00076829 & 0.3319452 & 1462.9986 & 2062.3557 & 2094.1005 \\
\hline 0.97 & $-67.6 \%$ & 0.15277365 & 4.52444219 & 0.97734963 & 0.3212595 & 301305.52 & 421168.63 & 426525.29 \\
\hline 0.98 & $-73.5 \%$ & 0.12473934 & 6.78430801 & 0.97715692 & 0.3211725 & 36505282 & 51024117 & 51672041 \\
\hline 0.99 & $-81.3 \%$ & 0.08820403 & 13.5684191 & 0.97714897 & 0.3211689 & $6.583 \mathrm{E}+17$ & $9.202 \mathrm{E}+17$ & $9.318 \mathrm{E}+17$ \\
\hline
\end{tabular}


Table 2. $G_{H 0} / \sqrt{\lambda_{f} \lambda_{p}}$ and $G_{H 0}$ for Hall Plates with $c m \neq 1 / 2 \wedge \lambda_{f}=\lambda_{p}=\sqrt{2}$. Each line of the table gives $G_{H 0} / \sqrt{\lambda_{f} \lambda_{p}}$ and $G_{H 0}$ for common mode fixed to the specific value of the leftmost column and $\lambda_{f}=\lambda_{p}=\sqrt{2} . \zeta_{3}, \zeta_{5}, \zeta_{6}$ are the corresponding parameters of (9), (11), (12). The data of this table is also plotted in graphical form in Figures $6(\mathrm{a})$-(d). The three rightmost columns specify the geometry of a rectangular Hall plate of Figure 3 (a) to achieve the specific common mode and $\lambda_{f}=\lambda_{p}=\sqrt{2}$. For each value of $\mathrm{cm}$ the noise relevant parameter $G_{H 0} / \sqrt{\lambda_{f} \lambda_{p}}$ is only negligibly smaller than in Table 1 , but the devices of Table 2 have the advantage of equal input and output resistances $R_{i n}=R_{\text {out }}=\sqrt{2} R_{\mathrm{sh}}$. SNRP loss is the reduction in $G_{H 0} / \sqrt{\lambda_{f} \lambda_{p}}$ compared to the overall maximum at $\mathrm{cm}=1 / 2$.

\begin{tabular}{|c|c|c|c|c|c|c|c|c|c|}
\hline $\mathrm{cm}$ & SNRP loss & $\frac{G_{H 0}}{\sqrt{\lambda_{f} \lambda_{p}}}$ & $G_{H 0}$ & $\zeta_{3}$ & $\zeta_{s}$ & $\zeta_{6}$ & $\ell / W$ & $b / \ell$ & $s / \ell$ \\
\hline 0.025 & $-76.7 \%$ & 0.109905743 & 0.155430192 & 1.002335535 & 1.013536098 & 21.31686947 & 1.4150 & 0.9630 & 0.0216 \\
\hline 0.05 & $-62.7 \%$ & 0.175994946 & 0.248894440 & 1.009521765 & 1.054276928 & 21.46355938 & 1.4171 & 0.9263 & 0.0427 \\
\hline 0.1 & $-43.6 \%$ & 0.266102531 & 0.376325808 & 1.040865803 & 1.218890953 & 21.99558033 & 1.4250 & 0.8547 & 0.0816 \\
\hline 0.15 & $-30.6 \%$ & 0.327057404 & 0.462529016 & 1.101607638 & 1.497368164 & 22.71303885 & 1.4352 & 0.7869 & 0.1139 \\
\hline 0.2 & $-21.2 \%$ & 0.371430098 & 0.525281482 & 1.202125012 & 1.893182063 & 23.44626216 & 1.4453 & 0.7237 & 0.1384 \\
\hline 0.25 & $-14.1 \%$ & 0.404827226 & 0.572512154 & 1.353665966 & 2.412091843 & 24.07721415 & 1.4538 & 0.6650 & 0.1558 \\
\hline 0.3 & $-8.8 \%$ & 0.430064674 & 0.608203294 & 1.567884442 & 3.06612838 & 24.56018366 & 1.4601 & 0.6099 & 0.1674 \\
\hline 0.35 & $-4.8 \%$ & 0.448649712 & 0.634486508 & 1.857742246 & 3.875385898 & 24.89883052 & 1.4645 & 0.5573 & 0.1749 \\
\hline 0.4 & $-2.1 \%$ & 0.461439423 & 0.652573890 & 2.238880866 & 4.867146861 & 25.1162362 & 1.4672 & 0.5066 & 0.1795 \\
\hline 0.45 & $-0.5 \%$ & 0.468934647 & 0.663173737 & 2.730987815 & 6.073054588 & 25.2360367 & 1.4687 & 0.4572 & 0.1820 \\
\hline 0.5 & $0.0 \%$ & 0.471404521 & 0.666666667 & 3.359160854 & 7.523945255 & 25.27414237 & 1.4692 & 0.4086 & 0.1827 \\
\hline 0.55 & $-0.5 \%$ & 0.468934647 & 0.663173737 & 4.155410812 & 9.240625886 & 25.2360367 & 1.4687 & 0.3609 & 0.1820 \\
\hline 0.6 & $-2.1 \%$ & 0.461439423 & 0.652573890 & 5.160361279 & 11.21821021 & 25.1162362 & 1.4672 & 0.3139 & 0.1795 \\
\hline 0.65 & $-4.8 \%$ & 0.448649712 & 0.634486508 & 6.42486482 & 13.40273689 & 24.89883052 & 1.4645 & 0.2677 & 0.1749 \\
\hline 0.7 & $-8.8 \%$ & 0.430064674 & 0.608203294 & 8.01016155 & 15.66453688 & 24.56018366 & 1.4601 & 0.2227 & 0.1674 \\
\hline 0.75 & $-14.1 \%$ & 0.404827226 & 0.572512154 & 9.981881174 & 17.78667319 & 24.07721415 & 1.4538 & 0.1792 & 0.1558 \\
\hline 0.8 & $-21.2 \%$ & 0.371430098 & 0.525281482 & 12.38457865 & 19.50401324 & 23.44626216 & 1.4453 & 0.1379 & 0.1384 \\
\hline 0.85 & $-30.6 \%$ & 0.327057404 & 0.462529016 & 15.16864015 & 20.61808402 & 22.71303885 & 1.4352 & 0.0992 & 0.1139 \\
\hline 0.9 & $-43.6 \%$ & 0.266102531 & 0.376325808 & 18.04556863 & 21.13200402 & 21.99558033 & 1.4250 & 0.0637 & 0.0816 \\
\hline 0.95 & $-62.7 \%$ & 0.175994946 & 0.248894440 & 20.35855931 & 21.26111604 & 21.46355938 & 1.4171 & 0.0310 & 0.0427 \\
\hline 0.975 & $-76.7 \%$ & 0.109905723 & 0.155430165 & 21.03217264 & 21.26719592 & 21.31686619 & 1.4150 & 0.0154 & 0.0216 \\
\hline
\end{tabular}

relies on conformal mapping theory introduced by [24] but it employs a novel way to derive the weak magnetic field limit. The numerical problems in the evaluation of the integrals are reduced via several transformations and closed form integrals given in the appendix. The agreement between the analytical theory and selected numerical simulation results is excellent. The set of Equations ((1), (22), (23), (27), (50), (54), (56)) gives the Hall output signal as an implicit function of electrically measurable quantities $\lambda_{f}, \lambda_{p}, \mathrm{~cm}$ irrespective of the device geometry. To the best of the author's knowledge, this work gives the most comprehensive closed form treatment of magnetic detectivity for a very general class of Hall 
Table 3. Comparison of analytical theory of this paper with finite element simulation for two Hall plates with $\mathrm{cm}=0.85$. The Hall plates are rectangular as in Figure 3(a). Both have a common mode equal to 0.85 . The first Hall plate has optimized sizes of contacts to maximize $G_{H 0} / \sqrt{\lambda_{f} \lambda_{p}}$, which leads to different input and output resistances $\lambda_{f, o p t} R_{\mathrm{sh}}, \lambda_{p, o p t} R_{\mathrm{sh}}$ with $\lambda_{f, \text { opt }}, \lambda_{p, \text { opt }}$ from Table 1 for $\mathrm{cm}=0.85$. The second Hall plate has $1.4 \%$ smaller SNRP $\left(=G_{H 0} / \sqrt{\lambda_{f} \lambda_{p}}\right)$ but with equal input and output resistances of $\sqrt{2}$ squares. The table compares values of these quantities obtained by our analytical theory with values obtained by finite element simulations (FEM), and it gives the differences of both.

\begin{tabular}{ccccccc}
\hline \multirow{2}{*}{ Quantity } & \multicolumn{3}{c}{ First Hall plate } & \multicolumn{3}{c}{ Second Hall plate } \\
\cline { 2 - 6 } & Theory & FEM & Difference & Theory & FEM & Difference \\
\hline$\ell / W$ & & 1.77012983 & & & 1.43517967 & \\
b $/ \ell$ & & 0.09203691 & & 0.09920905 & \\
$s / \ell$ & & 0.13541332 & & & 0.11390467 & \\
$c m$ & 0.85000 & 0.85000 & $0.0003 \%$ & 0.85000 & 0.84999 & $0.001 \%$ \\
$\lambda_{f}$ & 1.72559 & 1.72560 & $-0.001 \%$ & 1.41421 & 1.41419 & $0.001 \%$ \\
$\lambda_{p}$ & 1.27011 & 1.26983 & $0.022 \%$ & 1.41421 & 1.41399 & $0.016 \%$ \\
$G_{H 0}$ & 0.49096 & 0.49083 & $0.026 \%$ & 0.46253 & 0.46251 & $0.005 \%$ \\
$G_{H 0,67}$ & 0.13312 & 0.13325 & $-0.097 \%$ & 0.11091 & 0.11097 & $-0.058 \%$ \\
$G_{H 0,12}$ & 0.35784 & 0.35771 & $0.038 \%$ & 0.35162 & 0.35154 & $0.024 \%$ \\
$G_{H 0} / \sqrt{\lambda_{f} \lambda_{p}}$ & 0.33163 & 0.33158 & $0.015 \%$ & 0.32706 & 0.32708 & $-0.006 \%$ \\
$3 G_{H 0} / \sqrt{2 \lambda_{f} \lambda_{p}}$ & 0.70350 & 0.70339 & $0.015 \%$ & 0.69379 & 0.69384 & $-0.006 \%$ \\
$\lambda_{f} / \lambda_{p}$ & 1.35862 & 1.35892 & $-0.022 \%$ & 1.00000 & 1.00014 & $-0.014 \%$ \\
\hline
\end{tabular}

effect devices.

With this theory, it was shown that the SNR is only slightly impaired if the common mode output potential deviates moderately from half of the supply potential. Despite the lack in symmetry for $c m \neq 1 / 2$ it is possible to keep input and output resistances equal without significant further loss in SNR. In Appendix C, the theory was applied to optimize of Vertical Hall effect devices with three top contacts and one buried layer contact. There it was possible to specify geometries with sufficiently large contacts for practical use and with only moderate loss in SNR. Moreover, it was shown that for $c m \neq 1 / 2$ the magnetic sensitivity of both contacts on the axis of mirror symmetry is not the same.

The theory is so general that it also covers former results on Hall plates with four contacts having two perpendicular mirror symmetries [1] and also former results on Hall plates with three contacts having only single mirror symmetry [31]. However, the mathematical expressions for the Hall plate parameters from the present theory show no apparent similarity to former work in [1] [31]. 


\section{Conflicts of Interest}

The author declares no conflicts of interest regarding the publication of this paper.

\section{References}

[1] Ausserlechner, U. (2017) The Signal-to-Noise Ratio and a Hidden Symmetry of Hall Plates. Solid-State Electronics, 135, 14-23. https://doi.org/10.1016/j.sse.2017.06.007

[2] Popovic, R.S. (1984) The Vertical Hall-Effect Device. IEEE Electron Device Letters, 5, 357-358. https://doi.org/10.1109/EDL.1984.25945

[3] Maenaka, K. (1993) Piezoresistive Effect in Vertical Hall Cell. Electronics Letters, 29, 381-382. https://doi.org/10.1049/el:19930256

[4] Stoica, D. and Motz, M. (2013) A Dual Vertical Hall Latch with Direction Detection. 2013 Proceedings of the ESSCIRC (ESSCIRC), Bucharest, 16-20 September 2013. https://doi.org/10.1109/ESSCIRC.2013.6649110

[5] Francis, L.A. and Kirill, P. (Eds.) (2017) Chapter 7 Magnetic Sensors and Devices: Technologies and Applications. In: Magnetic Angle Sensors, CRC Press, Boca Raton, Florida.

[6] Ausserlechner, U. (2016) Hall Effect Devices with Three Terminals: Their Magnetic Sensitivity and Offset Cancellation Scheme. Journal of Sensors, 2016, Article ID 5625607. https://doi.org/10.1155/2016/5625607

[7] Tiger, C. and Juang, K.-C. (2014) CMOS SC-Spinning, Current-Feedback Hall Sensor for High Speed and Low Cost Applications. 2014 IEEE SENSORS, Valencia, 2-5 November 2014. https://doi.org/10.1109/ICSENS.2014.6985051

[8] Stoessel, Z. and Resch, M. (1993) Flicker Noise and Offset Suppression in Symmetric Hall Plates. Sensors and Actuators A: Physical, 37, 449-452. https://doi.org/10.1016/0924-4247(93)80076-S

[9] Versnel, W. (1978) Analysis of Symmetrical Van der Pauw Structures with Finite Contacts. Solid-State Electronics, 21, 1261-1268. https://doi.org/10.1016/0038-1101(78)90376-3

[10] Cornils, M., Doelle, M. and Paul, O. (2007) Sheet Resistance Determination Using Symmetric Structures with Contacts of Finite Size. IEEE Transactions on Electron Devices, 54, 2756-2761. https://doi.org/10.1109/TED.2007.906219

[11] Ausserlechner, U. (2016) Closed form Expressions for Sheet Resistance and Mobility from Van-der-Pauw Measurement on $90^{\circ}$ Symmetric Devices with Four Arbitrary Contacts. Solid-State Electronics, 116, 46-55.

https://doi.org/10.1016/j.sse.2015.11.030

[12] Versnel, W. (1981) Analysis of Symmetrical Hall Plates with Finite Contacts. Journal of Applied Physics, 52, 4659-4666. https://doi.org/10.1063/1.329347

[13] Ausserlechner, U. (2016) Simple Formula for Hall-Geometry Factor of Hall-Plates with $90^{\circ}$ Symmetry. UPB Science Bulletin Series A, 78, 275-282.

[14] Ausserlechner, U. (2016) A Method to Compute the Hall-Geometry Factor at Weak Magnetic Field in Closed Analytical Form. Electrical Engineering, 98, 189-206. https://doi.org/10.1007/s00202-015-0351-4

[15] Ausserlechner, U. (2017) Van-der-Pauw Measurement on Devices with Four Contacts and Two Orthogonal Mirror Symmetries. Solid-State Electronics, 133, 53-63. https://doi.org/10.1016/j.sse.2017.04.006

[16] Ausserlechner, U. (2004) Limits of Offset Cancellation by the Principle of Spinning 
Current Hall Probe. Proceedings of IEEE SENSORS, Vienna, Austria, 24-27 October 2004, 1117-1120.

[17] Cornils, M. (2009) Sheet Resistance and Hall Mobility Determination beyond von der Pauw. Vol. 12, MEMS-Engineering and Technology, $210 \mathrm{p}$.

[18] Cornils, M. and Paul, O. (2009) The Magnetic Calibration of Arbitrarily Shaped Hall Sensors in the Absence of Magnetic Fields. Proceedings of 2009 International Solid-State Sensors, Actuators and Microsystems Conference, Denver, CO, USA, 21-25 June 2009, 881-884.

[19] Homentcovschi, D. and Bercia R. (2018) Analytical Solution for the Electric Field in Hall Plates. Zeitschrift für angewandte Mathematik und Physik, 69, 97.

[20] Besse P.A., Schott, C. and Popovic, R.S. (1998) Analytical Study of Vertical Hall (VH)-Devices Using an Adapted Conform Mapping Technique. Technical Proceedings of the 1998 International Conference on Modelling and Simulation of Microsystems, Santa Clara, California, 6-8 April 1998, 660-665.

[21] http://functions.wolfram.com/EllipticIntegrals/EllipticF/16/02/01

[22] Strassacker, G. and Strassacker, P. (1993) Analytische und numerische Methoden der Feldberechnung. Teubner, Stuttgart, $94 \mathrm{p}$.

https://doi.org/10.1007/978-3-322-91229-9

[23] http://functions.wolfram.com/EllipticFunctions/JacobiSN/03/01/02/02/0001/

[24] Wick, R.F. (1954) Solution of the Field Problem of the Germanium Gyrator. Journal of Applied Physics, 25, 741-756. https://doi.org/10.1063/1.1721725

[25] Versnel, W. (1981) Analysis of a Circular Hall Plate with Equal Finite Contacts. Solid-State Electronics, 24, 63-68. https://doi.org/10.1016/0038-1101(81)90213-6

[26] Lippmann, H.-J. and Kuhrt, F. (1958) Der Geometrieeinfluß auf den transversalen magnetischen Widerstandseffekt bei rechteckförmigen Halbleiterplatten. Zeitschrift für Naturforschung A, 13, 462-474. https://doi.org/10.1515/zna-1958-0608

[27] Haeusler, J. (1966) Exakte Lösungen von Potentialproblemen beim Halleffekt durch Konforme Abbidungen. Solid-State Electronics, 9, 417-441. https://doi.org/10.1016/0038-1101(66)90156-0

[28] Prudnikov, A.P., Brychkov, Y.A. and Marichev, O.I. (1968) Integrals and Series. Vol. 1, 1.2.36.1 and 1.2.38.1, Gordon and Breach Science Publishers, New York, USA.

[29] Prudnikov, A.P., Brychkov, Y.A., and Marichev, O.I. (1968) Integrals and Series. Vol, 1, 1.2.35.1 and 1.2.39.1, Gordon and Breach Science Publishers, New York, USA.

[30] Prudnikov, A.P., Brychkov, Y.A. and Marichev, O.I. (1968) Integrals and Series. Vol, 1, 1.2.40.1 and 1.2.33.1, Gordon and Breach Science Publishers, New York, USA.

[31] Ausserlechner, U. (2018) An Analytical Theory of Hall-Effect Devices with Three Contacts. Open Physics Journal, 4, 14-42. https://doi.org/10.2174/1874843001804010014

[32] Borwein, J.M. and Borwein, P.B. (1986) Pi and the AGM. John Wiley \& Sons, Hoboken, NJ. 


\section{Appendix A}

Here are the definitions of some functions used in the text. The imaginary unit is denoted by $i=\sqrt{-1}$. The incomplete elliptic integral of the first order is defined as

$$
F(w, k)=\int_{0}^{w}\left(1-\alpha^{2}\right)^{-1 / 2}\left(1-k^{2} \alpha^{2}\right)^{-1 / 2} \mathrm{~d} \alpha .
$$

The complete integral of the first order is given by

$$
K(k)=F(1, k) \text {. }
$$

It holds $K(0)=\pi / 2$. Asymptotic limit $K(k \rightarrow 1)=\ln \left(4 / \sqrt{1-k^{2}}\right)$. We also use the common notation for the complementary elliptic integral

$$
K^{\prime}(k)=K\left(\sqrt{1-k^{2}}\right)
$$

Inversion of (A1) gives the Jacobi-sn function

$$
w=\operatorname{sn}(F(w, k), k) .
$$

From (A4) it follows $0=\operatorname{sn}(0, k)$ and $\pm 1=\operatorname{sn}( \pm K(k), k)$.

The modular lambda function is defined as the inverse of the monotonic function $K^{\prime}(k) / K(k)$ in the interval $0 \leq k \leq 1$

$$
L\left(K^{\prime}(k) / K(k)\right)=k^{2} .
$$

Further useful properties of this function are given in [15].

\section{Appendix B}

\section{B1. The $G$-Functions}

We introduce the following functions which appear in the calculation of the resistances at zero magnetic field.

$$
g(\zeta)=\frac{1}{\sqrt{\left|\left(\zeta^{2}-1\right)\left(\zeta^{2}-\zeta_{3}^{2}\right)\left(\zeta^{2}-\zeta_{5}^{2}\right)\left(\zeta^{2}-\zeta_{6}^{2}\right)\right|}}>0
$$

with $1 \leq \zeta_{3} \leq \zeta_{5} \leq \zeta_{6}$. For $n=0,1,2$

$$
\begin{gathered}
G_{n}^{\prime}=\int_{\zeta=0}^{1} \zeta^{n} g(\zeta) \mathrm{d} \zeta \\
\bar{G}_{n}=\int_{\zeta=1}^{\zeta_{3}} \zeta^{n} g(\zeta) \mathrm{d} \zeta \\
G_{n}=\int_{\zeta=\zeta_{3}}^{\zeta_{5}} \zeta^{n} g(\zeta) \mathrm{d} \zeta>0 \\
\overline{\bar{G}}_{n}=\int_{\zeta=\zeta_{5}}^{\zeta_{6}} \zeta^{n} g(\zeta) \mathrm{d} \zeta \\
G_{n}^{\prime \prime}=\int_{\zeta=\zeta_{6}}^{\infty} \zeta^{n} g(\zeta) \mathrm{d} \zeta
\end{gathered}
$$


The integrands of $G_{n}^{\prime}$ and $G_{n}^{\prime \prime}$ have only a single pole, whereas the integrands of $\bar{G}_{n}, G_{n}, \overline{\bar{G}}_{n}$ have two poles. All $G$-functions are positive. For $n=1$ these integrals can be solved explicitly by [28] (if we use the substitution $x=y^{2}$ in the integrand of [28]).

$$
\begin{aligned}
& \bar{G}_{1}=\frac{1}{\sqrt{\left(\zeta_{6}^{2}-\zeta_{3}^{2}\right)\left(\zeta_{5}^{2}-1\right)}} K^{\prime}\left(\sqrt{\frac{\zeta_{5}^{2}-\zeta_{3}^{2}}{\zeta_{6}^{2}-\zeta_{3}^{2}} \frac{\zeta_{6}^{2}-1}{\zeta_{5}^{2}-1}}\right) \\
& G_{1}=\frac{1}{\sqrt{\left(\zeta_{6}^{2}-\zeta_{3}^{2}\right)\left(\zeta_{5}^{2}-1\right)}} K\left(\sqrt{\frac{\zeta_{5}^{2}-\zeta_{3}^{2}}{\zeta_{6}^{2}-\zeta_{3}^{2}} \frac{\zeta_{6}^{2}-1}{\zeta_{5}^{2}-1}}\right)
\end{aligned}
$$

From Figure 4 it follows

$$
\overline{W_{2} W_{3}}=\overline{W_{5} W_{6}} \Leftrightarrow \bar{G}_{1}=\overline{\bar{G}}_{1}
$$

One can get (B9) by direct calculation of the integrals according to [29]. With [30] it holds

$$
\begin{gathered}
G_{1}^{\prime}=\frac{1}{\sqrt{\left(\zeta_{6}^{2}-\zeta_{3}^{2}\right)\left(\zeta_{5}^{2}-1\right)}} F\left(\frac{1}{\zeta_{3}} \sqrt{\frac{\zeta_{6}^{2}-\zeta_{3}^{2}}{\zeta_{6}^{2}-1}}, \sqrt{\frac{\zeta_{5}^{2}-\zeta_{3}^{2}}{\zeta_{6}^{2}-\zeta_{3}^{2}} \frac{\zeta_{6}^{2}-1}{\zeta_{5}^{2}-1}}\right), \\
G_{1}^{\prime \prime}=\frac{1}{\sqrt{\left(\zeta_{6}^{2}-\zeta_{3}^{2}\right)\left(\zeta_{5}^{2}-1\right)}} F\left(\sqrt{\frac{\zeta_{5}^{2}-1}{\zeta_{6}^{2}-1}}, \sqrt{\frac{\zeta_{5}^{2}-\zeta_{3}^{2}}{\zeta_{6}^{2}-\zeta_{3}^{2}} \frac{\zeta_{6}^{2}-1}{\zeta_{5}^{2}-1}}\right) .
\end{gathered}
$$

and with the addition theorems for elliptic integrals in [21] one gets

$$
G_{1}^{\prime} \pm G_{1}^{\prime \prime}=\frac{1}{\sqrt{\left(\zeta_{6}^{2}-\zeta_{3}^{2}\right)\left(\zeta_{5}^{2}-1\right)}} F\left(\frac{\zeta_{3} \zeta_{5} \pm \zeta_{6}}{\zeta_{5} \pm \zeta_{3} \zeta_{6}}, \sqrt{\frac{\zeta_{5}^{2}-\zeta_{3}^{2}}{\zeta_{6}^{2}-\zeta_{3}^{2}} \frac{\zeta_{6}^{2}-1}{\zeta_{5}^{2}-1}}\right)
$$

The physical meaning of $G_{1}^{\prime} / G_{1}$ and $G_{1}^{\prime \prime} / G_{1}$ is the size of the folded contacts $\overline{W_{0} W_{2}}$ and $\overline{W_{6} W_{\infty}}$ on the axis of symmetry in Figure 4. Only in the particular symmetric case $\mathrm{cm}=1 / 2$ it holds $\overline{W_{0} W_{2}}=\overline{W_{6} W_{\infty}}$ and therefore $G_{1}^{\prime}=G_{1}^{\prime \prime}$. Yet in general $G_{1}^{\prime} \neq G_{1}^{\prime \prime}$. A comparison of (B8) and (B12) gives $G_{1}^{\prime}+G_{1}^{\prime \prime} \leq G_{1}$, where the equal sign is only valid in the two degenerate cases $\zeta_{3} \rightarrow 1$ (which means $\mathrm{cm} \rightarrow 0$ ) and $\zeta_{5} \rightarrow \zeta_{6}$ (which means $\mathrm{cm} \rightarrow 1$ ). In both cases $\lambda_{p}=0$ (see (27)).

Another identity between $G$-functions can be proven with Figure B1. It shows a mapping from the upper half of the $\zeta$-plane onto the interior of a polygon in the $w^{\prime}$-plane. The valid Schwartz-Christoffel transformation is

$$
w^{\prime}=C^{\prime} \int_{0}^{\zeta} \frac{\sqrt{\zeta^{2}-\zeta_{3}^{2}}}{\sqrt{\zeta^{2}-1} \sqrt{\zeta^{2}-\zeta_{5}^{2}} \sqrt{\zeta^{2}-\zeta_{6}^{2}}} \mathrm{~d} \zeta
$$

With (B13) we compute the following segments

$$
\overline{W_{0}^{\prime} W_{2}^{\prime}}=\left|C^{\prime}\right| \int_{0}^{1} \frac{\left|\zeta^{2}-\zeta_{3}^{2}\right|}{\sqrt{\left|\left(\zeta^{2}-1\right)\left(\zeta^{2}-\zeta_{3}^{2}\right)\left(\zeta^{2}-\zeta_{5}^{2}\right)\left(\zeta^{2}-\zeta_{6}^{2}\right)\right|}} \mathrm{d} \zeta=\left|C^{\prime}\right|\left(\zeta_{3}^{2} G_{0}^{\prime}-G_{2}^{\prime}\right)
$$




$$
\begin{aligned}
& \overline{W_{3}^{\prime} W_{5}^{\prime}}=\left|C^{\prime}\right| \int_{\zeta_{3}}^{\zeta_{5}} \frac{\left|\zeta^{2}-\zeta_{3}^{2}\right|}{\sqrt{\left|\left(\zeta^{2}-1\right)\left(\zeta^{2}-\zeta_{3}^{2}\right)\left(\zeta^{2}-\zeta_{5}^{2}\right)\left(\zeta^{2}-\zeta_{6}^{2}\right)\right|}} \mathrm{d} \zeta=\left|C^{\prime}\right|\left(G_{2}-\zeta_{3}^{2} G_{0}\right) \\
& \overline{W_{6}^{\prime} W_{\infty}^{\prime}}=\left|C^{\prime}\right| \int_{\zeta_{6}}^{\infty} \frac{\left|\zeta^{2}-\zeta_{3}^{2}\right|}{\sqrt{\left|\left(\zeta^{2}-1\right)\left(\zeta^{2}-\zeta_{3}^{2}\right)\left(\zeta^{2}-\zeta_{5}^{2}\right)\left(\zeta^{2}-\zeta_{6}^{2}\right)\right|}} \mathrm{d} \zeta=\left|C^{\prime}\right|\left(G_{2}^{\prime \prime}-\zeta_{3}^{2} G_{0}^{\prime \prime}\right)
\end{aligned}
$$

According to Figure B1 it holds $\overline{W_{0}^{\prime} W_{2}^{\prime}}+\overline{W_{3}^{\prime} W_{5}^{\prime}}=\overline{W_{6}^{\prime} W_{\infty}^{\prime}}$. This gives

$$
\zeta_{3}^{2}\left(G_{0}^{\prime}-G_{0}+G_{0}^{\prime \prime}\right)=G_{2}^{\prime}-G_{2}+G_{2}^{\prime \prime} .
$$

A similar mapping is shown in Figure B2. It is given by

$$
w^{\prime \prime}=C^{\prime \prime} \int_{0}^{\zeta} \frac{\sqrt{\zeta^{2}-1}}{\sqrt{\zeta^{2}-\zeta_{3}^{2}} \sqrt{\zeta^{2}-\zeta_{5}^{2}} \sqrt{\zeta^{2}-\zeta_{6}^{2}}} \mathrm{~d} \zeta
$$

with the lengths

$$
\begin{aligned}
& \overline{W_{1}^{\prime \prime} W_{2}^{\prime \prime}}=2\left|C^{\prime \prime}\right| \int_{0}^{1} \frac{1-\zeta^{2}}{\sqrt{\left|\left(\zeta^{2}-1\right)\left(\zeta^{2}-\zeta_{3}^{2}\right)\left(\zeta^{2}-\zeta_{5}^{2}\right)\left(\zeta^{2}-\zeta_{6}^{2}\right)\right|}} \mathrm{d} \zeta=2\left|C^{\prime \prime}\right|\left(G_{0}^{\prime}-G_{2}^{\prime}\right) \\
& \overline{W_{3}^{\prime \prime} W_{5}^{\prime \prime}}=\left|C^{\prime \prime}\right| \int_{\zeta_{3}}^{\zeta_{5}} \frac{\zeta^{2}-1}{\sqrt{\left|\left(\zeta^{2}-1\right)\left(\zeta^{2}-\zeta_{3}^{2}\right)\left(\zeta^{2}-\zeta_{5}^{2}\right)\left(\zeta^{2}-\zeta_{6}^{2}\right)\right|}} \mathrm{d} \zeta=\left|C^{\prime \prime}\right|\left(G_{2}-G_{0}\right) \\
& \overline{W_{6}^{\prime \prime} W_{7}^{\prime \prime}}=2\left|C^{\prime \prime}\right| \int_{\zeta_{6}}^{\infty} \frac{\zeta^{2}-1}{\sqrt{\left|\left(\zeta^{2}-1\right)\left(\zeta^{2}-\zeta_{3}^{2}\right)\left(\zeta^{2}-\zeta_{5}^{2}\right)\left(\zeta^{2}-\zeta_{6}^{2}\right)\right|}} \mathrm{d} \zeta=2\left|C^{\prime \prime}\right|\left(G_{2}^{\prime \prime}-G_{0}^{\prime \prime}\right)
\end{aligned}
$$

With Figure $\mathrm{B} 2$ it holds $\overline{W_{1}^{\prime \prime} W_{2}^{\prime \prime}}+2 \overline{W_{3}^{\prime \prime} W_{5}^{\prime \prime}}=\overline{W_{6}^{\prime \prime} W_{7}^{\prime \prime}}$. This gives

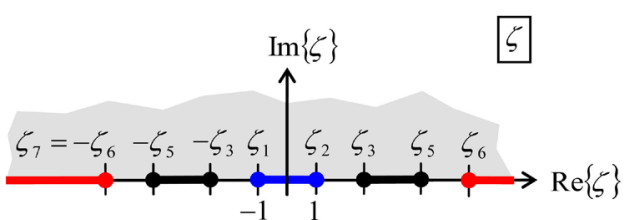

(a)

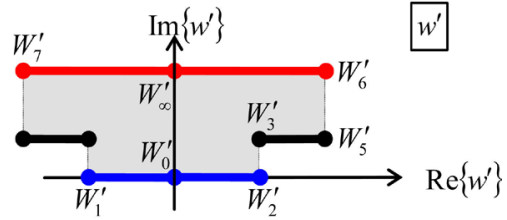

(b)

Figure B1. (a) Is a representation of the Hall plate with single mirror symmetry in the upper half of the $\zeta$-plane with contacts on the real axis-it is identical to Figure 3(b). (b) Shows the same Hall-plate after conformal transformation (B13).

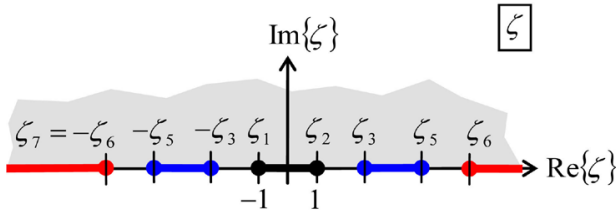

(a)

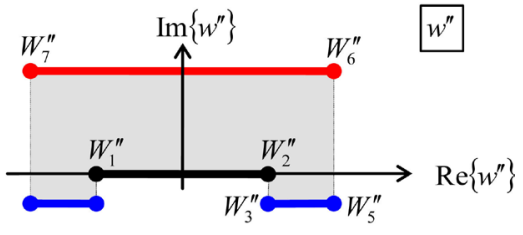

(b)

Figure B2. (a) Is a representation of the Hall plate with single mirror symmetry in the upper half of the $\zeta$-plane with contacts on the real axis-it is identical to Figure $3(\mathrm{~b})$. (b) Shows the same Hall-plate after conformal transformation (B18). 


$$
G_{0}^{\prime}-G_{0}+G_{0}^{\prime \prime}=G_{2}^{\prime}-G_{2}+G_{2}^{\prime \prime} .
$$

The combination of (B17) and (B22) gives two remarkable identities.

$$
\begin{aligned}
& G_{0}=G_{0}^{\prime}+G_{0}^{\prime \prime} \\
& G_{2}=G_{2}^{\prime}+G_{2}^{\prime \prime}
\end{aligned}
$$

\section{B2. The $H$-Functions}

In the calculation of the Hall signal the following function appears.

$$
h(\zeta)=g(\zeta) \ln \left|\frac{1-\zeta}{1+\zeta} \frac{\zeta_{3}+\zeta}{\zeta_{3}-\zeta} \frac{\zeta_{5}-\zeta}{\zeta_{5}+\zeta} \frac{\zeta_{6}+\zeta}{\zeta_{6}-\zeta}\right|
$$

with $1<\zeta_{3}<\zeta_{5}<\zeta_{6} . h(\zeta)$ has four zeros.

$$
\begin{gathered}
h(0)=h\left(\zeta_{13,0}\right)=h\left(\zeta_{35,0}\right)=h\left(\zeta_{56,0}\right)=0 \\
\zeta_{1}<\zeta_{13,0}=\sqrt{\zeta_{3}-\zeta_{5}+\zeta_{3} \zeta_{5}+\zeta_{6}-\zeta_{3} \zeta_{6}+\zeta_{5} \zeta_{6}-\mathrm{sqrt}} / \sqrt{2}<\zeta_{3} \\
\zeta_{3}<\zeta_{35,0}=\sqrt{\frac{\zeta_{3} \zeta_{5} \zeta_{6}+\zeta_{3} \zeta_{6}-\zeta_{3} \zeta_{5}-\zeta_{5} \zeta_{6}}{-1+\zeta_{3}-\zeta_{5}+\zeta_{6}}}<\zeta_{5} \\
\zeta_{5}<\zeta_{56,0}=\sqrt{\zeta_{3}-\zeta_{5}+\zeta_{3} \zeta_{5}+\zeta_{6}-\zeta_{3} \zeta_{6}+\zeta_{5} \zeta_{6}+\mathrm{sqrt}} / \sqrt{2}<\zeta_{6}
\end{gathered}
$$

with

$$
\text { sqrt }=\sqrt{-4 \zeta_{3} \zeta_{5} \zeta_{6}+\left(\zeta_{3}-\zeta_{5}+\zeta_{6}+\zeta_{3} \zeta_{5}-\zeta_{3} \zeta_{6}+\zeta_{5} \zeta_{6}\right)^{2}}
$$

We define for $n=0,1,2$

$$
\begin{aligned}
& H_{n}^{\prime}=\int_{\zeta=0}^{1} \zeta^{n} h(\zeta) \mathrm{d} \zeta \\
& \bar{H}_{n}=\int_{\zeta=1}^{\zeta_{3}} \zeta^{n} h(\zeta) \mathrm{d} \zeta \\
& H_{n}=\int_{\zeta_{3}}^{\zeta_{5}} \zeta^{n} h(\zeta) \mathrm{d} \zeta \\
& \overline{\bar{H}}_{n}=\int_{\zeta=\zeta_{5}}^{\zeta_{6}} \zeta^{n} h(\zeta) \mathrm{d} \zeta \\
& H_{n}^{\prime \prime}=\int_{\zeta=\zeta_{6}}^{\infty} \zeta^{n} h(\zeta) \mathrm{d} \zeta
\end{aligned}
$$

The integrands of $H_{n}^{\prime}$ and $H_{n}^{\prime \prime}$ are singular only at one end of the integration interval, whereas the integrands of $\bar{H}_{n}, H_{n}, \overline{\bar{H}}_{n}$ are singular at both ends. The integrands of $H_{n}^{\prime}$ and $H_{n}^{\prime \prime}$ have no zeros, yet the integrands of $\bar{H}_{n}, H_{n}, \overline{\bar{H}}_{n}$ have one zero. Therefore, the signs of $\bar{H}_{n}, H_{n}, \overline{\bar{H}}_{n}$ are not obvious, whereas $H_{n}^{\prime}<0$ and $H_{n}^{\prime \prime}>0$.

There are several relations between these integrals such as $\bar{H}_{0}=\overline{\bar{H}}_{0}$ and $\bar{H}_{2}=\overline{\bar{H}}_{2}$ (given without proof). For the purpose of this work we need relations 
of $H$-functions with index 1 . To this end we consider the conformal transformation of Figure B3 given by

$$
w^{\prime \prime \prime}=\int_{0}^{\zeta} \frac{C_{1}^{\prime \prime \prime}(\zeta-1)^{1 / 2+m}\left(\zeta-\zeta_{6}\right)^{1 / 2-m} d \zeta}{(\zeta+1)^{1 / 2+m}\left(\zeta-\zeta_{3}\right)^{1 / 2+m}\left(\zeta-\zeta_{5}\right)^{1 / 2-m}\left(\zeta+\zeta_{6}\right)^{1 / 2-m}\left(\zeta+\zeta_{5}\right)^{1 / 2+m}\left(\zeta+\zeta_{3}\right)^{1 / 2-m}}+C_{0}^{\prime \prime \prime}
$$

with $m=\beta / \pi$. In the limit of small $m$ it holds

$$
\begin{aligned}
& \overline{W_{7}^{\prime \prime \prime} W_{6}^{\prime \prime \prime}}=\left|C_{1}^{\prime \prime \prime}\right|\left|\int_{-\infty}^{-\zeta_{6}}+\int_{\zeta_{6}}^{\infty} \frac{(\zeta-1)\left(\zeta-\zeta_{6}\right)\left[1+m \ln \left(\frac{\zeta-1}{\zeta+1} \frac{\zeta+\zeta_{3}}{\zeta-\zeta_{3}} \frac{\zeta-\zeta_{5}}{\zeta+\zeta_{5}} \frac{\zeta+\zeta_{6}}{\zeta-\zeta_{6}}\right)\right] \mathrm{d} \zeta \mid}{\left(\zeta^{2}-1\right)^{1 / 2}\left(\zeta^{2}-\zeta_{3}^{2}\right)^{1 / 2}\left(\zeta^{2}-\zeta_{5}^{2}\right)^{1 / 2}\left(\zeta^{2}-\zeta_{6}^{2}\right)^{1 / 2}}\right| \\
& =\left|C_{1}^{\prime \prime \prime}\right|\left[G_{2}^{\prime \prime}+\left(1+\zeta_{6}\right) G_{1}^{\prime \prime}+\zeta_{6} G_{0}^{\prime \prime}-m\left(H_{2}^{\prime \prime}+\left(1+\zeta_{6}\right) H_{1}^{\prime \prime}+\zeta_{6} H_{0}^{\prime \prime}\right)\right. \\
& \left.+G_{2}^{\prime \prime}-\left(1+\zeta_{6}\right) G_{1}^{\prime \prime}+\zeta_{6} G_{0}^{\prime \prime}+m\left(H_{2}^{\prime \prime}-\left(1+\zeta_{6}\right) H_{1}^{\prime \prime}+\zeta_{6} H_{0}^{\prime \prime}\right)\right] \\
& \overline{W_{5}^{\prime \prime \prime} W_{3}^{\prime \prime \prime}}=\left|C_{1}^{\prime \prime \prime}\right|\left|\int_{\zeta_{3}}^{\zeta_{5}}(\zeta-1)\left(\zeta-\zeta_{6}\right)(g(\zeta)+m h(\zeta)) \mathrm{d} \zeta\right| \\
& =\left|C_{1}^{\prime \prime \prime}\right|\left[-G_{2}+\left(1+\zeta_{6}\right) G_{1}-\zeta_{6} G_{0}+m\left(-H_{2}+\left(1+\zeta_{6}\right) H_{1}-\zeta_{6} H_{0}\right)\right]
\end{aligned}
$$

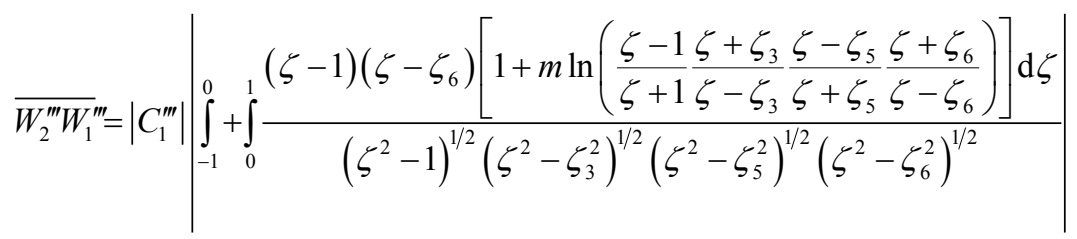

$$
\begin{aligned}
& =\left|C_{1}^{\prime \prime \prime}\right|\left[G_{2}^{\prime}+\left(1+\zeta_{6}\right) G_{1}^{\prime}+\zeta_{6} G_{0}^{\prime}-m\left(H_{2}^{\prime}+\left(1+\zeta_{6}\right) H_{1}^{\prime}+\zeta_{6} H_{0}^{\prime}\right)\right. \\
& \left.+G_{2}^{\prime}-\left(1+\zeta_{6}\right) G_{1}^{\prime}+\zeta_{6} G_{0}^{\prime}+m\left(H_{2}^{\prime}-\left(1+\zeta_{6}\right) H_{1}^{\prime}+\zeta_{6} H_{0}^{\prime}\right)\right] \\
& \overline{W_{8}^{\prime \prime \prime} W_{10}^{\prime \prime \prime}}=\left|C_{1}^{\prime \prime \prime}\right|\left|\int_{-\zeta_{5}}^{-\zeta_{3}}(\zeta-1)\left(\zeta-\zeta_{6}\right)(g(\zeta)+m h(\zeta)) \mathrm{d} \zeta\right| \\
& =\left|C_{1}^{\prime \prime \prime}\right|\left[G_{2}+\left(1+\zeta_{6}\right) G_{1}+\zeta_{6} G_{0}-m\left(H_{2}+\left(1+\zeta_{6}\right) H_{1}+\zeta_{6} H_{0}\right)\right]
\end{aligned}
$$

With Figure $\mathrm{B} 3$ it holds $\overline{W_{8}^{\prime \prime \prime} W_{10}^{\prime \prime \prime}}=\overline{W_{7}^{\prime \prime \prime} W_{6}^{\prime \prime \prime}}+\overline{W_{5}^{\prime \prime \prime} W_{3}^{\prime \prime \prime}+} \overline{W_{2}^{\prime \prime \prime} W_{1}^{\prime \prime \prime}}$. At vanishing magnetic field this is fulfilled for $m^{0}$ terms due to (B23), (B24). Also the $m^{1}$ terms at small magnetic field must be identical. This gives

$$
H_{1}=H_{1}^{\prime}+H_{1}^{\prime \prime} \text {. }
$$

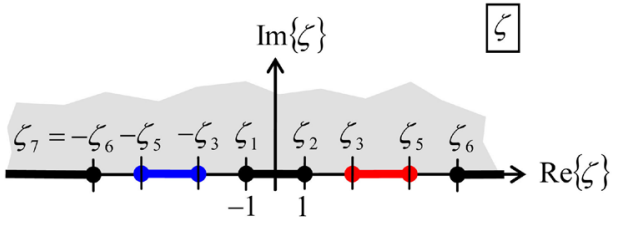

(a)

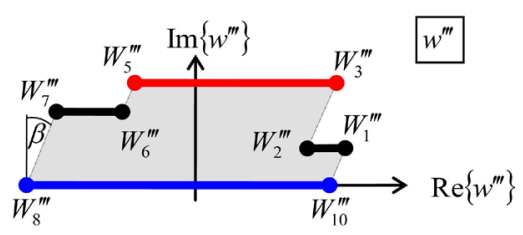

(b)

Figure B3. (a) Is a representation of the Hall plate with single mirror symmetry in the upper half of the $\zeta$-plane with contacts on the real axis-it is identical to Figure 3(b). (b) Shows the same Hall-plate after conformal transformation (B36). 


\section{B3. Numerical Evaluation of the Integrals}

The numerical evaluation of the integrals (B2-B6) and (B31-B35) may get tricky for $\zeta_{3} \rightarrow 1$ (which means $\mathrm{cm} \rightarrow 0$ ), $\zeta_{5} \rightarrow \zeta_{6}$ (which means $\mathrm{cm} \rightarrow 1$ ), and $\zeta_{3} \rightarrow \zeta_{5}$ (which means small partial contacts). The situation improves if we transform the integrals in the following way, which is explained exemplarily for $\overline{\bar{H}}_{n}(n=0,1,2)$. This integral extends from $\zeta_{5}$ to $\zeta_{6}$ with poles of the integrand at both ends. We split up the integration interval into two parts $\left(\zeta_{5}, \zeta_{56,0}\right)$ and $\left(\zeta_{56,0}, \zeta_{6}\right)$, because then we have only a single pole of the integrand in each of these intervals. Next we substitute the integration variables: for the lower interval we set $\zeta=\zeta_{5} \cosh \alpha$, and for the upper interval we set $\zeta=\zeta_{6} \cos \beta$. (Alternatively one could also substitute $\zeta=\zeta_{5} / \cos \alpha$ and/or $\zeta=\zeta_{6} / \cosh \beta$.) This gives

$$
\begin{aligned}
& \overline{\bar{H}}_{n}=\zeta_{5}^{n} \int_{\alpha=0}^{\operatorname{arccoshyp} \frac{\zeta_{56,0}}{\zeta_{5}}} \cosh ^{n} \alpha \frac{\ln \left(\frac{\cosh \alpha-1}{\cosh \alpha+1} \frac{\zeta_{5} \cosh \alpha-1}{\zeta_{5} \cosh \alpha+1} \frac{\zeta_{5} \cosh \alpha+\zeta_{5} \cosh \alpha-\zeta_{3}}{\zeta_{6}+\zeta_{5} \cosh \alpha}\right)}{\sqrt{\zeta_{5}^{2} \cosh ^{2} \alpha-1} \sqrt{\zeta_{5}^{2} \cosh ^{2} \alpha-\zeta_{3}^{2}} \sqrt{\zeta_{6}^{2}-\zeta_{5}^{2} \cosh ^{2} \alpha}} \mathrm{d} \alpha \\
& +\zeta_{6}^{n} \int_{\beta=0}^{\arccos \frac{\zeta_{6,0}}{\zeta_{6}}} \cos ^{n} \beta \frac{\ln \left(\frac{\cos \beta+1}{\cos \beta-1} \frac{\zeta_{6} \cos \beta-1}{\zeta_{6} \cos \beta+1} \frac{\zeta_{6} \cos \beta+\zeta_{6} \cos \beta-\zeta_{3}}{\zeta_{6} \cos \beta-\zeta_{5}} \frac{\zeta_{6} \cos \beta+\zeta_{5}}{\zeta_{6}^{2} \cos ^{2} \beta-1} \sqrt{\zeta_{6}^{2} \cos ^{2} \beta-\zeta_{3}^{2}} \sqrt{\zeta_{6}^{2} \cos ^{2} \beta-\zeta_{5}^{2}}\right.}{\sqrt{\zeta^{2}}} \mathrm{~d} \beta
\end{aligned}
$$

Thus, we avoided the poles in the denominators. Only the arguments of the logarithms have poles, which are easier to deal with by numerical integration routines. For the $\overline{\bar{G}}_{n}$ functions we simply skip the logarithms in (B42), and then we have no singularities at all. For the functions $H_{n}^{\prime}, H_{n}^{\prime \prime}, G_{n}^{\prime}, G_{n}^{\prime \prime}$ we do not need to split up the original integration interval. Then the integration intervals become $(0, \pi / 2)$ or $(0, \infty)$. The numerical integration routines of MATHEMATICA can handle the singularities in the $H$-integrals, when we choose the integration method Double Exponential, however, the maximum recursion limit and the working precision for numerical computations also have to be increased sufficiently.

\section{Appendix C}

Here we look for Vertical Hall effect devices of Figure 1(c) with large contacts and large SNRP. The geometrical parameters $\ell_{a}, \ell_{b}, \ell_{c}, \ell, d$ of these devices are labeled in Figure C1(a). In Figure C1(b) the geometry is scaled, and in Figure $\mathrm{C} 1(\mathrm{c})$ it is mapped onto the upper half of the $t$-plane. These transformations are

$$
\begin{gathered}
q=\tilde{z} / \ell, \\
t=\operatorname{sn}(2 q K(\kappa), \kappa), \\
\tilde{w}=-1 /(\kappa t),
\end{gathered}
$$

with the parameter $\kappa$ given by the aspect ratio of the Hall tub

$$
d / \ell=K^{\prime}(\kappa) /(2 K(\kappa)) \Leftrightarrow \kappa=\sqrt{L(2 d / \ell)} .
$$

For the contacts we get 


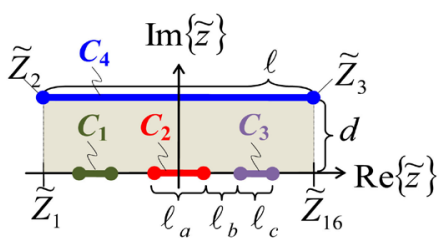

(a)

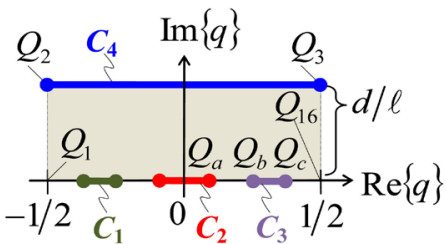

(b)

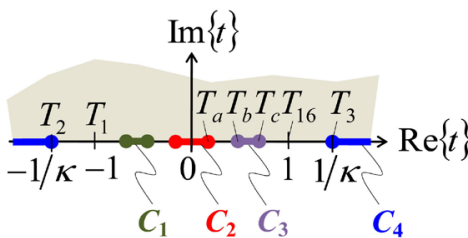

(c)

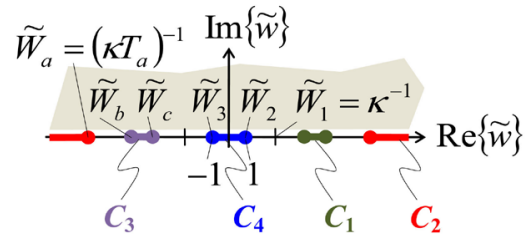

(d)

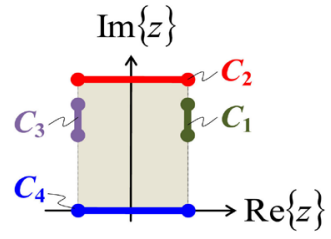

(e)

Figure C1. (a)-(d) Mapping of a Vertical Hall effect device with four contacts onto the upper half of the $\tilde{w}$-plane. In (a) contact $C_{4}$ extends along the entire length of the Hall tub, because in silicon BiCMOS technology it is made from the n-buried layer, which cannot be patterned differently than the Hall tub. When moving from contact $C_{1}$ over $C_{2}, C_{3}$ to $C_{4}$ the Hall effect region is on the left hand side. Contact $C_{2}$ is centered in the $\tilde{z}, q, t$-planes and it is at infinity in the $\tilde{w}$-plane. The device in the $\tilde{w}$-plane of Figure $\mathrm{C} 1(\mathrm{~d})$ is identical with the device in the $\zeta$-plane in Figure 3(b). (e) Mapping from the $\tilde{w}$-plane onto the original device in Figure 3(a).

$$
\begin{gathered}
T_{a}=\operatorname{sn}\left(\left(\ell_{a} / \ell\right) K(\kappa), \kappa\right), \\
T_{b}=\operatorname{sn}\left(\left(\ell_{a} / \ell+2 \ell_{b} / \ell\right) K(\kappa), \kappa\right), \\
T_{c}=\operatorname{sn}\left(\left(\ell_{a} / \ell+2 \ell_{b} / \ell+2 \ell_{c} / \ell\right) K(\kappa), \kappa\right) .
\end{gathered}
$$

A comparison of Figure C1(c) with Figure 3(b) gives

$$
\begin{aligned}
& \zeta_{3}=-\tilde{W}_{c}=1 /\left(\kappa T_{c}\right), \\
& \zeta_{5}=-\tilde{W}_{b}=1 /\left(\kappa T_{b}\right), \\
& \zeta_{6}=-\tilde{W}_{a}=1 /\left(\kappa T_{a}\right) .
\end{aligned}
$$

Inserting (C4-C7) into (C8-C10) gives three relations between the parameters $\zeta_{3}, \zeta_{5}, \zeta_{6}$ and the geometrical parameters of the device in Figure $\mathrm{C1}$ (a), which can be solved for $\ell_{a}, \ell_{b}, \ell_{c}$.

$$
\begin{gathered}
\ell_{a}=\ell F\left(\kappa^{-1} \zeta_{6}^{-1}, \kappa\right) / K(\kappa) \\
\ell_{b}=\ell\left[F\left(\kappa^{-1} \zeta_{5}^{-1}, \kappa\right)-F\left(\kappa^{-1} \zeta_{6}^{-1}, \kappa\right)\right] /(2 K(\kappa)) \\
\ell_{c}=\ell\left[F\left(\kappa^{-1} \zeta_{3}^{-1}, \kappa\right)-F\left(\kappa^{-1} \zeta_{5}^{-1}, \kappa\right)\right] /(2 K(\kappa))
\end{gathered}
$$

Before we turn to the case of general common mode, we study the special case $\mathrm{cm}=1 / 2$. Such a device may have a circular shape as in Figure C2. The two horizontal contacts have half aperture angle $\alpha_{1}$ and the two vertical contacts have half aperture angle $\alpha_{2}$. The centers of the contacts are on the $\operatorname{Re}\left\{z^{\prime}\right\}$ - and $\operatorname{Im}\left\{z^{\prime}\right\}$-axes; with this symmetry the common mode is $1 / 2$. This device can also be mapped to the upper half of the $w^{\prime}$-plane via the Möbius transformation 


$$
w^{\prime}=\frac{-i}{\tan \left(\alpha_{2} / 2\right)} \frac{z^{\prime}+i}{z^{\prime}-i} .
$$

The mappings of $Z_{a}^{\prime} \rightarrow W_{a}^{\prime}, \quad Z_{b}^{\prime} \rightarrow W_{b}^{\prime}$, and $Z_{c}^{\prime} \rightarrow W_{c}^{\prime}$ give

$$
\begin{gathered}
W_{a}^{\prime}=\frac{\cos \alpha_{2}+1}{\cos \alpha_{2}-1}, \\
W_{b}^{\prime}=\frac{\sin \alpha_{2}}{\cos \alpha_{2}-1} \frac{1+\cos \alpha_{1}+\sin \alpha_{1}}{1+\cos \alpha_{1}-\sin \alpha_{1}} \\
W_{c}^{\prime}=\frac{\sin \alpha_{2}}{\cos \alpha_{2}-1} \frac{1+\cos \alpha_{1}-\sin \alpha_{1}}{1+\cos \alpha_{1}+\sin \alpha_{1}} .
\end{gathered}
$$

For $\mathrm{cm}=1 / 2$ the $\tilde{w}$-plane in Figure $\mathrm{C} 1(\mathrm{~d})$ is identical to the $w^{\prime}$-plane in Figure C2(b). Inserting (C15-C17) into (C8-C10) gives (15). The impedance of the device of Figure $\mathrm{C} 2$ is computed by the transformations $z \rightarrow w \rightarrow t \rightarrow q$ in Figures C3(a)-(d).

$$
\begin{gathered}
w=\left(\frac{1-z^{2}}{1+z^{2}}\right)^{2} \\
t=\frac{A-w}{C+\kappa_{2} w} \\
A=1-\left(\cos \alpha_{1} \sin \alpha_{2}\right)^{-1} \\
C=\kappa_{2}(A-2) \\
\kappa_{2}=\frac{\cos \alpha_{1}-\sin \alpha_{2}}{\cos \alpha_{1}+\sin \alpha_{2}} \\
q=F\left(t, \kappa_{2}\right)
\end{gathered}
$$

Equations (C19-C22) define a Möbius transformation. The number of squares between the vertical contacts (the ones with half aperture angle $\alpha_{2}$ ) is

$$
\lambda_{2}=\frac{2 K\left(\kappa_{2}\right)}{K^{\prime}\left(\kappa_{2}\right)}=\frac{K^{\prime}\left(\left(1-\kappa_{2}\right) /\left(1+\kappa_{2}\right)\right)}{K\left(\left(1-\kappa_{2}\right) /\left(1+\kappa_{2}\right)\right)}=\frac{K^{\prime}\left(\sin \alpha_{2} / \cos \alpha_{1}\right)}{K\left(\sin \alpha_{2} / \cos \alpha_{1}\right)},
$$

where we used [32] and $K^{\prime}(k)=K\left(k^{\prime}\right)$ for the second identity in (C24). For the number of squares between the horizontal contacts (with half aperture angle $\alpha_{1}$ )

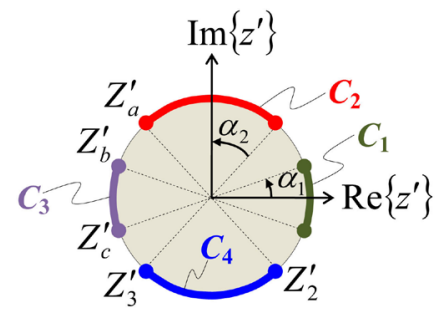

(a)

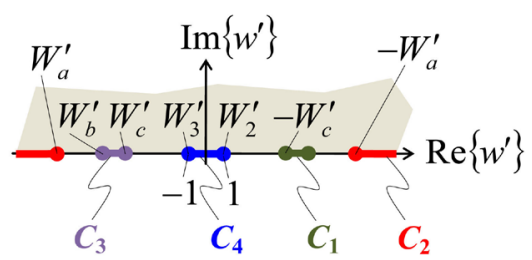

(b)

Figure C2. Mapping of a circular Hall plate with four contacts and $\mathrm{cm}=1 / 2$ onto the upper half of the $w^{\prime}$-plane. When moving from contact $C_{1}$ over $C_{2}, C_{3}$ to $C_{4}$ the Hall effect region is on the left hand side. 


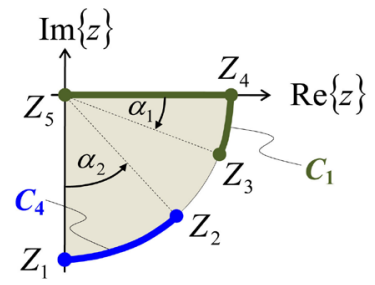

(a)

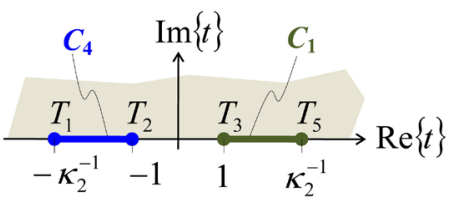

(c)

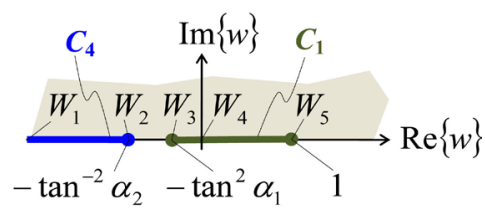

(b)

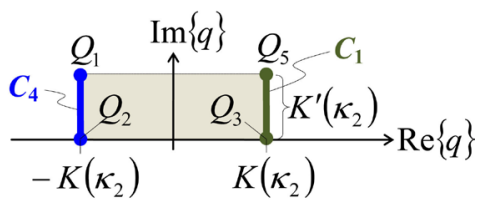

(d)

Figure C3. Mapping of a quarter of a circular Hall plate with four contacts and $\mathrm{cm}=1 / 2$ onto a rectangle in the $q$-plane to compute the number of squares between the vertical contacts of the device in Figure C2(a).

we only have to swap indices 1 and 2 .

$$
\lambda_{1}=\frac{K^{\prime}\left(\sin \alpha_{1} / \cos \alpha_{2}\right)}{K\left(\sin \alpha_{1} / \cos \alpha_{2}\right)}
$$

We can solve (C24, C25) for the half aperture angles with the help of the modular lambda function and insert this into (C15-C17), insert this again into (C8-C10) and finally into (C11-C13). With the abbreviations $L_{1}=L\left(\lambda_{1}\right)$, $L_{2}=L\left(\lambda_{2}\right)$ we get

$$
\begin{gathered}
\ell_{a}=\frac{\ell}{K(\kappa)} F\left(\frac{1}{\kappa} \frac{\left(\sqrt{1-L_{1} L_{2}}-\sqrt{1-L_{2}}\right)^{2}}{L_{2}\left(1-L_{1}\right)}, \kappa\right) \\
\ell_{b}=\frac{\ell}{2 K(\kappa)} F\left(\frac{1}{\kappa} \frac{\sqrt{1-L_{1} L_{2}}-\sqrt{1-L_{2}}}{\sqrt{L_{2}} \sqrt{1-L_{1}}} \frac{\sqrt{L_{1}} \sqrt{1-L_{2}}-\sqrt{1-L_{1} L_{2}}+\sqrt{1-L_{1}}}{\sqrt{L_{1}} \sqrt{1-L_{2}}+\sqrt{1-L_{1} L_{2}}-\sqrt{1-L_{1}}}, \kappa\right)-\frac{\ell_{a}}{2} \\
\ell_{c}=\frac{\ell}{2 K(\kappa)} F\left(\frac{1}{\kappa} \frac{\sqrt{1-L_{1} L_{2}}-\sqrt{1-L_{2}}}{\sqrt{L_{2}} \sqrt{1-L_{1}}} \frac{\sqrt{L_{1}} \sqrt{1-L_{2}}+\sqrt{1-L_{1} L_{2}}+\sqrt{1-L_{1}}}{-\sqrt{L_{1}} \sqrt{1-L_{2}}+\sqrt{1-L_{1} L_{2}}+\sqrt{1-L_{1}}}, \kappa\right)-\ell_{b}-\frac{\ell_{a}}{2}
\end{gathered}
$$

This gives the geometrical parameters of the device in Figure C1(a) for the case $\mathrm{cm}=1 / 2$ as a function of the numbers of squares $\lambda_{1}, \lambda_{2}$. According to [1] the maximum signal to noise ratio for fixed power (SNRP) is achieved for $\lambda_{1}=\lambda_{2}=\sqrt{2}$ and this means $L_{1}=L_{2}=3-2 \sqrt{2}$ [15]. Inserting this into (C26-C28) gives the relative lengths $\ell_{a} / \ell, \ell_{b} / \ell, \ell_{c} / \ell$ as functions of the aspect ratio $d / \ell$ of the Hall tub (see (C4)).

$$
\begin{gathered}
\ell_{a} \rightarrow \ell F\left(\kappa^{-1} \zeta_{6, \max }^{-1}, \kappa\right) / K(\kappa), \\
\ell_{b} \rightarrow \ell F\left(\kappa^{-1} \zeta_{5, \max }^{-1}, \kappa\right) /(2 K(\kappa))-\ell_{a} / 2, \\
\ell_{c} \rightarrow \ell F\left(\kappa^{-1} \zeta_{3, \max }^{-1}, \kappa\right) /(2 K(\kappa))-\ell_{b}-\ell_{a} / 2,
\end{gathered}
$$


with the numbers $\zeta_{3, \max }, \zeta_{5, \max }, \zeta_{6, \max }$ from (58-60). For $\ell=20 \mu \mathrm{m}$ and $d=5 \mu \mathrm{m}$ one gets $\ell_{a}=0.254 \mu \mathrm{m}, \ell_{b}=0.302 \mu \mathrm{m}, \ell_{c}=0.556 \mu \mathrm{m}$. In practice these values are smaller than the feature size of many CMOS technologies, and they give rise to large electric fields when operated at typical supply voltages of around $2 \mathrm{~V}$. Large electric fields lead to velocity saturation and electrical non-linearity and local self-heating, which reduce the efficiency of the spinning current Hall probe scheme [16]. Since the tub depth $d$ cannot be increased in standard technologies we can only play with the length $\ell$. According to Figure C4 the length $\ell$ should be small in order to make the contact spacing $\ell_{b}$ large. However, the arguments in the incomplete elliptic integrals in (C29-C31) must not exceed 1 for real valued solutions. Equation (C31) gives the most stringent requirement:

$$
\ell \geq \frac{2 K(-1+2 \sqrt{2}-2 \sqrt{2-\sqrt{2}})}{K^{\prime}(-1+2 \sqrt{2}-2 \sqrt{2-\sqrt{2}})} d \cong 1.22004 d
$$

For maximum SNRP the length of the Hall tub must be at least $22 \%$ larger than its depth. Moreover, the outer contacts should reach towards the end of the tub $\ell_{a}+2 \ell_{b}+2 \ell_{c}=\ell$. This follows from (C31) when the first argument of the incomplete elliptic integral equates 1 . For $d=5 \mu \mathrm{m}$ one gets $\ell_{a}=0.506 \mu \mathrm{m}$, $\ell_{b}=0.628 \mu \mathrm{m}, \ell_{c}=2.170 \mu \mathrm{m}$ and $\ell=6.100 \mu \mathrm{m}$. In the following we will see that it is possible to increase the contacts even further if we depart from $\mathrm{cm}=1 / 2$.

In the general case of Hall effect devices with different input and output resistances it is advantageous to operate them in a stacked way according to Figure C5. Two identical devices are connected in series such that the entire supply current flows through $C_{1}-C_{3}$ of one device and through $C_{2}-C_{4}$ of the other device. Then a spinning Hall probe scheme can be implemented, where input and output

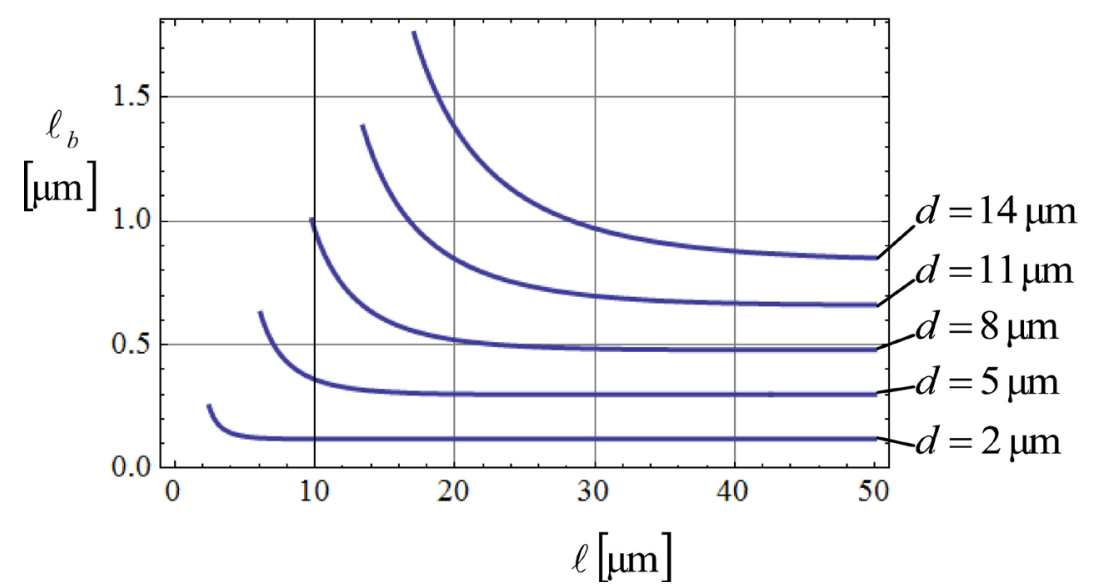

Figure C4. Contact spacing $\ell_{b}$ as a function of tub length $\ell$ and depth $d$ for Vertical Hall effect devices of Figure C1(a) with maximum SNRP, i.e. with $\mathrm{cm}=1 / 2 \wedge \lambda_{1}=\lambda_{2}=\sqrt{2}$ computed with (C27) in the valid range of (C32). $\ell_{b}$ is small and $\ell_{a}, \ell_{c}$ (not shown) are similarly small. 


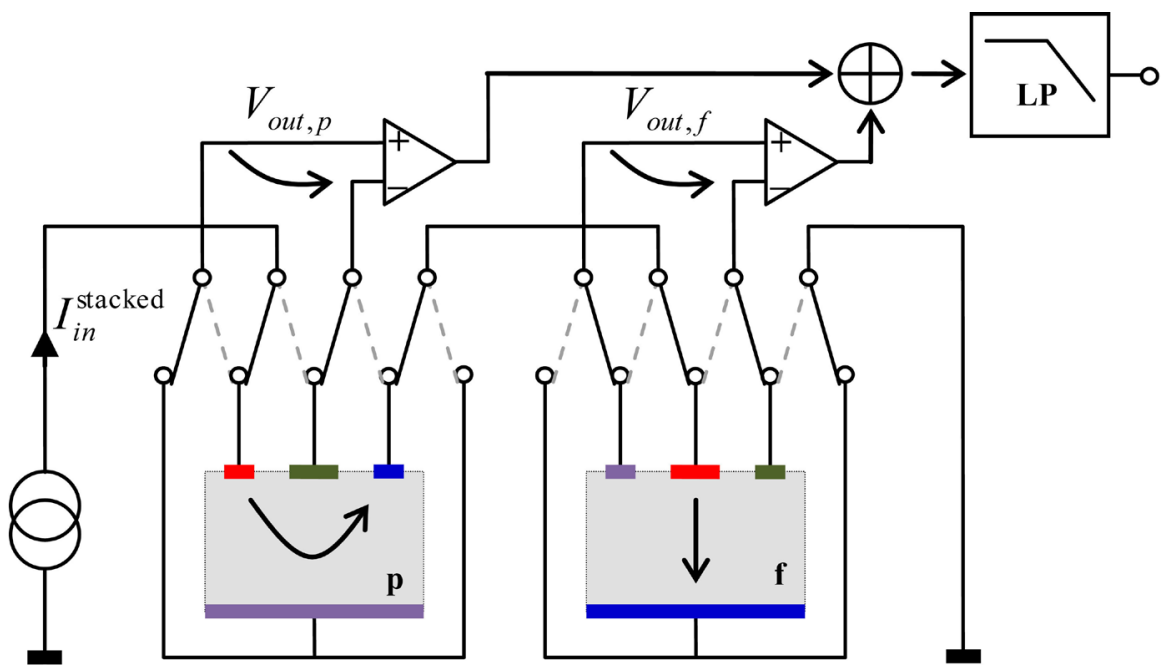

Figure C5. Two identical Vertical Hall effect devices of Figure C1(a) are operated in a stack: supply wise the devices are connected in series. The device at higher potential is in p-phase with current flowing across its axis of mirror symmetry, while the device at lower potential is in f-phase with current flowing along its axis of mirror symmetry. The output voltages are tapped at different common mode potentials, amplified by operational amplifiers, whose outputs are added and low pass filtered. The switches implement a spinning current Hall probe scheme where each device toggles between $\mathrm{p}$ and $\mathrm{f}$ mode a frequency well above the low pass corner frequency. If all phases of the spinning scheme last equally long and the current is constant during all phases the offset (zero point) error of the output vanishes in linear electrostatic theory. In practice a tiny residual offset is left: it corresponds to an offset equivalent field of about $10-100 \mu \mathrm{T}$ in silicon technology. This residual offset is caused by small asymmetries in combination with electrical nonlinearities such as velocity saturation, self-heating, and thermoelectric voltages caused by the Seebeck effect. The residual offset increases with the magnitude of the electric field in the devices.

terminals of both devices are swapped synchronously while a constant supply current is forced through them, and the output voltages at the remaining terminals are added for both devices and both operating phases. This cancels out offset errors and low frequency 1/f-noise [8] [16]. The sum of resistances of both devices is nominally the same in both operating phases: $R_{i n}^{\text {stacked }}=\left(\lambda_{f}+\lambda_{p}\right) R_{s h}$. Thus, at the fixed supply current $I_{i n}^{\text {stacked }}$ also the total supply voltage across both devices is nominally constant in both operating phases. The sum of output voltages of both devices in each operating phase is $2 \mu_{H} R_{s h} G_{H 0} I_{\text {in }}^{\text {stacked }} B_{\perp}$ and the thermal noise voltage in this sum of output voltages is $\sqrt{4 k_{b} T\left(\lambda_{f}+\lambda_{p}\right) R_{s h} E N B W}$, with Boltzmann's constant $k_{b}$, the absolute temperature $T$, and the effective noise bandwidth $E N B W$ of the signal path. This gives the signal-to-noise ratio

$$
S N R^{\text {stacked }}=\mu_{H} \frac{B_{\perp}}{\sqrt{4 k_{b} T E N B W}} \frac{G_{H 0}}{\left(\lambda_{f}+\lambda_{p}\right) / 2} \frac{V_{\text {in }}^{\text {stacked }}}{\sqrt{R_{\text {in }}^{\text {stacked }}}}
$$

The last factor in (C33) is equal to the square-root of the power dissipated in both devices. Equation (C33) gives the SNR of stacked Hall effect devices related to the dissipated power. This SNRP of the stack uses the arithmetic mean 
$\left(\lambda_{f}+\lambda_{p}\right) / 2$ whereas the SNRP of single devices discussed above used the geometric mean $\sqrt{\lambda_{f} \lambda_{p}}$ (see also (8) in [1]). Both are identical for symmetric devices with $\lambda_{f}=\lambda_{p}$, but in the general case the arithmetic mean is larger than the geometric mean and therefore it holds $S N R P^{\text {stacked }} \leq S N R P$. The maxima of both SNRP-s are also identical and so the maximum value of $2 G_{H 0} /\left(\lambda_{f}+\lambda_{p}\right)$ is again $\sqrt{2} / 3$. Hence, we should look for large contacts and contact spacing with $3 \sqrt{2} G_{H 0} /\left(\lambda_{f}+\lambda_{p}\right)$ close to 1 . Thereby, $\lambda_{f}, \lambda_{p}, \mathrm{~cm}$ may vary arbitrarily. Results of a numerical search are given in Table 4 and Figure C6. The first lines in Table 4 show that there exist Vertical Hall devices with low noise and with geometries that avoid unduly small contacts and contact spacing.

Table 4. Collection of Vertical Hall effect device geometries of Figure C1(a). The depth of the Hall tub is $d=5 \mu \mathrm{m}$ and the three lengths $\ell_{a}, \ell_{b}, \ell_{c}$ were varied in integer multiples of $0.5 \mu \mathrm{m}$. The length of the Hall tub was varied as $\ell=4,5,6,7 \mu \mathrm{m}$. The SNRP loss is given for stacked operation according to Figure C5-it is equal to $3 \sqrt{2} G_{H 0} /\left(\lambda_{f}+\lambda_{p}\right)-1$. The one but rightmost column gives the distance between the outer contacts and the edges of the Hall tub. The rightmost column gives the smallest length. The table is sorted according to $\min \left(\ell_{a}, \ell_{b}, \ell_{c}\right)$ and SNRP loss. The table comprises only data of the 50 devices with largest contact lengths and largest SNRP. Figure C6 gives a plot of the data in $2^{\text {nd }}$ and $3^{\text {rd }}$ columns for all 138 possible combinations of contact lengths.

\begin{tabular}{|c|c|c|c|c|c|c|c|c|c|c|c|}
\hline$\#$ & SNRP loss & $\mathrm{cm}$ & $\lambda_{f}$ & $\lambda_{p}$ & $G_{H 0}$ & $\ell_{[\mu \mathrm{m}]}$ & $\begin{array}{ll}\ell_{a} & {[\mu \mathrm{m}]}\end{array}$ & $\begin{array}{ll}\ell_{b} & {[\mu \mathrm{m}]}\end{array}$ & $\begin{array}{c}\ell_{c} \\
{[\mu \mathrm{m}]}\end{array}$ & $\begin{array}{c}\left(\ell-\ell_{a}\right) / 2-\ell_{b}-\ell_{c} \\
{[\mu \mathrm{m}]}\end{array}$ & $\begin{array}{c}\min \left(\ell_{a}, \ell_{b}, \ell_{c}\right) \\
{[\mu \mathrm{m}]}\end{array}$ \\
\hline 1 & $-4.1 \%$ & 0.483 & 1.173 & 1.716 & 0.6532 & 7 & 1 & 1 & 2 & 0 & 1 \\
\hline 2 & $-4.5 \%$ & 0.486 & 1.175 & 1.751 & 0.6589 & 7 & 1 & 1 & 1.5 & 0.5 & 1 \\
\hline 3 & $-5.9 \%$ & 0.535 & 1.253 & 1.918 & 0.7035 & 6 & 1 & 1 & 1.5 & 0 & 1 \\
\hline 4 & $-6.0 \%$ & 0.497 & 1.181 & 1.872 & 0.6763 & 7 & 1 & 1 & 1 & 1 & 1 \\
\hline 5 & $-6.9 \%$ & 0.540 & 1.255 & 1.983 & 0.7104 & 6 & 1 & 1 & 1 & 0.5 & 1 \\
\hline 6 & $-9.0 \%$ & 0.529 & 1.056 & 1.868 & 0.6274 & 7 & 1.5 & 1 & 1.5 & 0.25 & 1 \\
\hline 7 & $-10.6 \%$ & 0.537 & 1.059 & 1.968 & 0.6380 & 7 & 1.5 & 1 & 1 & 0.75 & 1 \\
\hline 8 & $-10.6 \%$ & 0.451 & 1.187 & 2.133 & 0.6996 & 7 & 1 & 1.5 & 1.5 & 0 & 1 \\
\hline 9 & $-11.3 \%$ & 0.600 & 1.370 & 2.225 & 0.7511 & 5 & 1 & 1 & 1 & 0 & 1 \\
\hline 10 & $-11.7 \%$ & 0.455 & 1.188 & 2.203 & 0.7056 & 7 & 1 & 1.5 & 1 & 0.5 & 1 \\
\hline 11 & $-12.7 \%$ & 0.586 & 1.134 & 2.130 & 0.6718 & 6 & 1.5 & 1 & 1 & 0.25 & 1 \\
\hline 12 & $-14.6 \%$ & 0.567 & 0.974 & 2.005 & 0.5999 & 7 & 2 & 1 & 1.5 & 0 & 1 \\
\hline 13 & $-15.1 \%$ & 0.508 & 1.262 & 2.486 & 0.7502 & 6 & 1 & 1.5 & 1 & 0 & 1 \\
\hline 14 & $-15.7 \%$ & 0.571 & 0.975 & 2.071 & 0.6048 & 7 & 2 & 1 & 1 & 0.5 & 1 \\
\hline 15 & $-16.9 \%$ & 0.499 & 1.065 & 2.360 & 0.6705 & 7 & 1.5 & 1.5 & 1 & 0.25 & 1 \\
\hline 16 & $-20.0 \%$ & 0.628 & 1.052 & 2.324 & 0.6365 & 6 & 2 & 1 & 1 & 0 & 1 \\
\hline 17 & $-20.4 \%$ & 0.432 & 1.192 & 2.679 & 0.7259 & 7 & 1 & 2 & 1 & 0 & 1 \\
\hline 18 & $-21.5 \%$ & 0.604 & 0.912 & 2.195 & 0.5750 & 7 & 2.5 & 1 & 1 & 0.25 & 1 \\
\hline 19 & $-23.6 \%$ & 0.539 & 0.979 & 2.573 & 0.6392 & 7 & 2 & 1.5 & 1 & 0 & 1 \\
\hline 20 & $-28.3 \%$ & 0.639 & 0.863 & 2.373 & 0.5469 & 7 & 3 & 1 & 1 & 0 & 1 \\
\hline 21 & $-0.2 \%$ & 0.519 & 1.363 & 1.453 & 0.6627 & 7 & 0.5 & 0.5 & 1 & 1.75 & 0.5 \\
\hline 22 & $-0.2 \%$ & 0.530 & 1.418 & 1.369 & 0.6554 & 6 & 0.5 & 0.5 & 1.5 & 0.75 & 0.5 \\
\hline 23 & $-0.3 \%$ & 0.497 & 1.340 & 1.324 & 0.6259 & 7 & 0.5 & 0.5 & 1.5 & 1.25 & 0.5 \\
\hline 24 & $-0.3 \%$ & 0.523 & 1.408 & 1.323 & 0.6416 & 6 & 0.5 & 0.5 & 2 & 0.25 & 0.5 \\
\hline
\end{tabular}




\section{Continued}

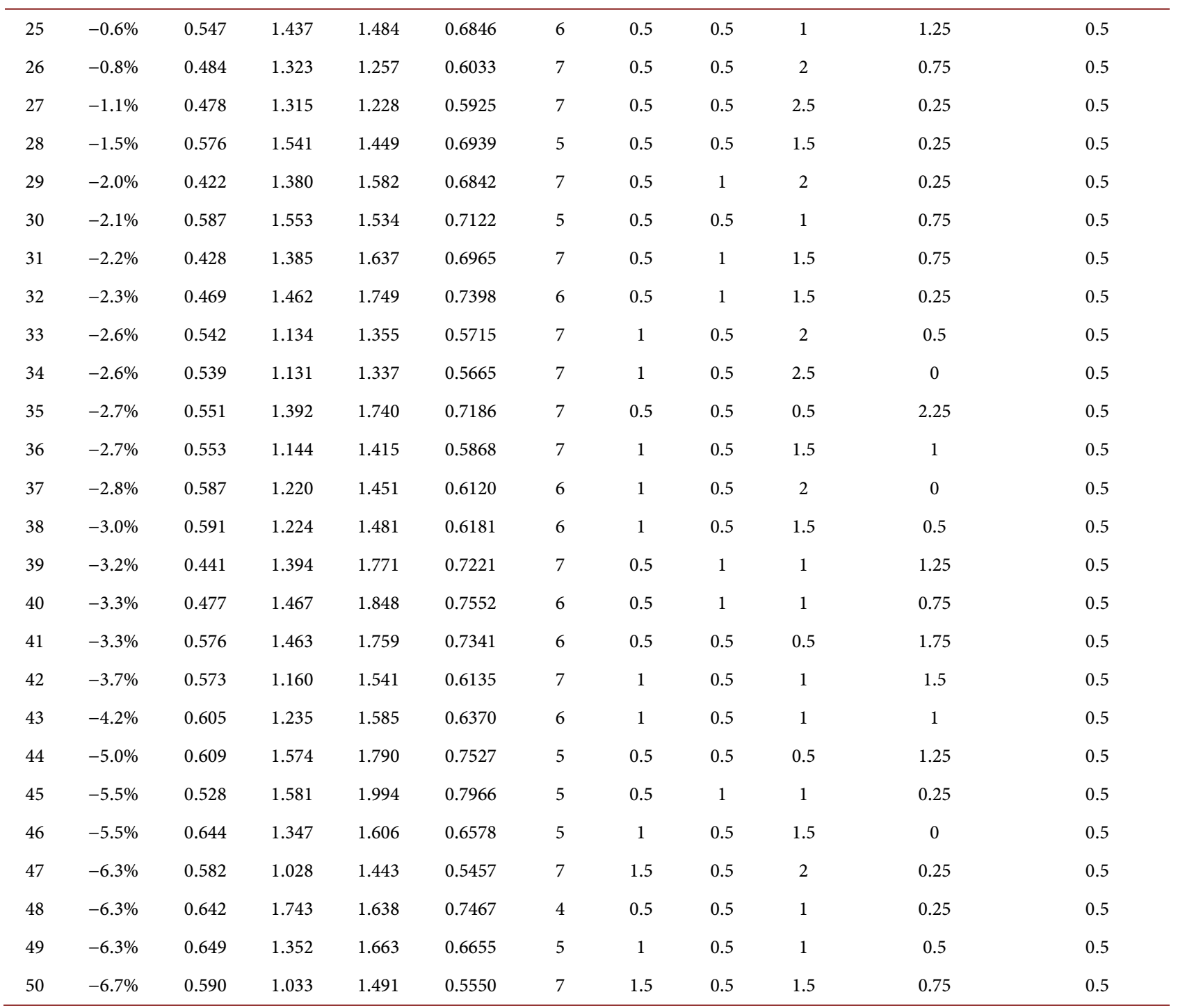

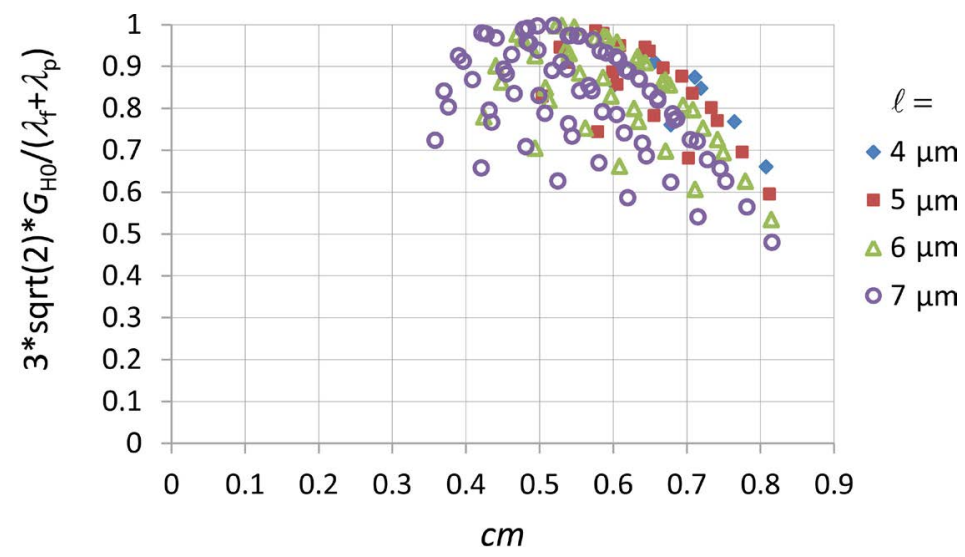

Figure C6. Scatter plot of normalized SNRP versus common mode $\mathrm{cm}$ of Vertical Hall effect devices like in Figure C1(a). The plot contains all 138 geometries explained in the caption of Table 4. 50 devices with large contact length and large SNRP are explicitly given in Table 4. The envelope of the scatter plot ressembles the graph in Figure 6(a). Due to the discretization in $0.5 \mu \mathrm{m}$ steps for $\ell_{a}, \ell_{b}, \ell_{c}$, points corresponding to short tubs tend to be at $\mathrm{cm}>0.5$. 